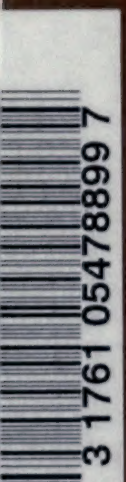

\title{
S\&M A 534
}


Digitized by the Internet Archive in 2007 with funding from Microsoft Corporation 


\title{
Condensed Catalogue
}

\author{
OF \\ BETHLEHEM \\ STRUCTURAL \\ SHAPES $\pi$
}

MANUEAOTURED BY

Bethlehem Steer Company

1) BETHLFHEM, PA. 击

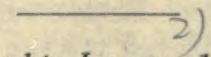

Revised to January, 1922.

GENERAL OFFICES:

At the Works, Bethlehem, Pa.

\section{SALES OFFICES:}

Boston, 141 Milk Street.

New York, 25 Broadway.

Philadel.PHa, Morris Building.

Atr.anta, Healey Building.

Pittsburgh, First National Bank Building.

Cleveland, Guardian Building.

Crucaco, Peoples Gas Building.

San Francisco, Monadnock Building.

Copyright, 1922, Bethlehem Steel Company. 


\section{NOTICE.}

This catalogue gives dimensions, weights and properties of all Bethlehem Structural Shapes, revised to date.

All Bethlehem I Beams shown in this catalogue are now available, also all Bethlehem $\mathrm{H}$ Columns and Bethlehem Supplementary Columns.

All Bethlehem Girder Beams shown in this catalogue will be available April 1, 1922, after which date the previous sections of girder beams will be discontinued.

Weights of all sections include weights of fillets, in conformity with the established practice adopted by the Association of American Steel Manufacturers.

The sections are numbered throughout the tables for convenience in identification and ordering.

A Handbook containing full tables of safe loads and other information is in course of preparation. The safe load tables given in the 1911 Catalogue are correct as printed for all sections that have not been superseded.

Bethlehem, Pa.

Bethlehem Steel Company. January, 1922. 


\section{EXPLANATORY NOTES.}

Large additional mill capacity for the manufacture of wide flange structural shapes has made the production of new sections practical where it had been found desirable for the improvement of the previously established line of Bethlehem Structural Shapes. Several new I beam and column sections were recently added, information concerning which has been published. A further desirable improvement has now been made in modifying Bethlehem Girder Beam sections to provide more than one weight of a section. All additional and modified sections to date are shown in this catalogue, together with all previous sections that have not been superseded.

Bethlehem Girder Beams. In general three weights are provided for each section, the middleweights corresponding to the previous sections of Bethlehem Girder Beams from G8 to $\mathrm{G} 24 \mathrm{a}$, inclusive. The lighter and heavier weights of each section provide opportunity for greater economy in the selection of sizes for any required purpose. To accomplish this, slight modifications in profile have been made in all girder sections from G8 to G24 a, inclusive. Simplification in the production of the larger girder sections, G26, G28 and G30, has been obtained by changing the profiles to an extent permitting all variations in weights of each girder to be procured from the same set of rolls.

Attention is called to the manner in which the variation in weight of the new girders is obtained by increasing or decreasing the thickness of the flange as well as that of the web, shown in Fig. 3, instead of obtaining the entire variation in weight by increasing or decreasing the thickness of the web only. This method was first successfully applied to the three new I beam sections B18 a, B22 and B24 b, and has now been extended to all the girder beams.

This method of rolling, on which patents are pending, permits obtaining a variation in Section Modulus practically proportional to the variation in weight. Thus G12, 61 lbs., compared with G12, 55.5 lbs., has an increase of 7.2 in Section Modulus, or about 10 per cent increase in modulus, due to the increase of $5.5 \mathrm{lbs}$. in weight, which is about 10 per cent increase in weight also. The increase or decrease in flange thickness is made at the outside of the flange, thereby slightly varying the depth of the beam; but the total variation in depth from minimum to maximum is not more than the usual allowable tolerance in depth necessary in any method of rolling beams.

The flanges of all gircer beams have a bevel of $8 \frac{1}{3}$ per cent, or a slope of 1 in 12. 
Bethlehem I Beams. No changes have been made in Bethlehem I Beam sections, and the list of sizes is the same as previously, except that the three new I beam sections, recently added, viz.: B18 a, B22 and B24 b, are included. These three new beam sections are increased by the same method as explained for Bethlehem Girder Beams, and their flanges have the same bevel of $8 \frac{1}{3}$ per cent, or a slope of 1 in 12 .

All other Bethlehem I Beams are increased, as shown in Fig. 1, by spreading the main rolls, which adds an equal amount to the thickness of the web and to the width of the flanges, all other dimensions remaining unchanged. The bevel of the flanges is 9 per cent.

Bethlehem Columns. Four new weights have been added to the series of $12^{\prime \prime} \mathrm{H}$ Columns, and two new weights to the series of $10^{\prime \prime} \mathrm{H}$ Columns. Tables are given of properties of $14^{\prime \prime} \mathrm{H}$ Columns reinforced with cover plates, when columns are required of greater sectional area than the largest 14" $\mathrm{H}$ Column rolled.

All Supplementary Column Sections, which were recently added, are given in this catalogue.

Bethlehem column sections are increased, as shown in Fig. 2, by spreading both sets of rolls; the thickness of the web and the width of the flanges are increased equally, the thickness of the flanges being hincreased a proportionate amount.

Flanges of all column sections have a slope of 2 per cent.
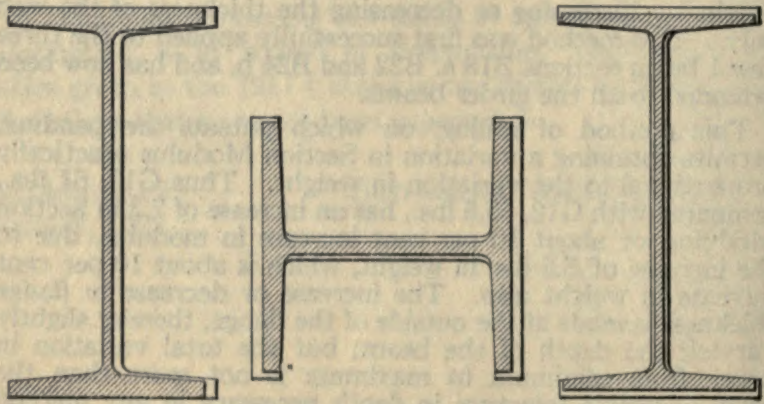

Fig. 1

Fig. 2

Fig. 3 
Bending Factors. To aid in the selection of columns subjected to direct loads and also to bending, produced by eccentric loads or by other means, bending factors are given, which furnish a convenient and practical method of converting the bending moment into an equivalent direct central load.

$\mathrm{P}^{\prime}$-centrally applied load, in lbs.

$\mathrm{P}^{\prime \prime}$-eccentric load, in lbs.

$\mathrm{m}$-bending moment, in inch lbs., produced by $\mathrm{P}^{\prime \prime}$.

$\mathrm{P}^{\prime \prime \prime}$-an equivalent central load, in lbs., producing the same maximum stress as bending moment.

P -total equivalent load on column, in lbs.

f -fiber stress, lbs. per sq. in., due to bending.

$\mathrm{S}$-section modulus about axis of bending.

A -area of section, square inches.

$\mathrm{k}$-bending factor $=\mathrm{A} \div \mathrm{S}$.

Then, $f=\frac{m}{S}$, and, by assumption, $\frac{P^{\prime \prime \prime}}{A}-f$.

But as $k-\frac{A}{S}$, then $f-\frac{m k}{A}-\frac{P^{\prime \prime \prime}}{A}$, and $P^{\prime \prime \prime}-m k$.

Therefore, $\mathrm{P}-\mathrm{P}^{\prime}+\mathrm{P}^{\prime \prime}+\mathrm{P}^{\prime \prime \prime}-\mathrm{P}^{\prime}+\mathrm{P}^{\prime \prime}+\mathrm{mk}$.

Hence, if the bending moment, in inch lbs., is multiplied by the bending factor, $\mathrm{k}$, the product is an equivalent central load on the column producing the same compressive stress as the bending moment.

Material. All Bethlehem Structural Shapes are of open hearth steel exclusively, conforming to Manufacturers' Standard specifications; and also to American Railway Engineering and Maintenance of Way Association specifications. Material complying with any other standard specifications may be furnished by special arrangement.

\section{GENERAL CONDITIONS.}

Shapes will be cut to ordered length within an allowable variation either way, as follows: Bethlehem I Beams $8^{\prime \prime}$ to $24^{\prime \prime}$, inclusive, within $8 / 8$ inch; all other sections, within $1 / 2$ inch. For cuttıng with less variation, or to exact length, an extra price is charged.

Sections are furnished only at catalogue weight. Shapes may have an allowable variation of $21 / 2$ per cent from nominal section. 
BETHLEHEM STEEL COMPANY.

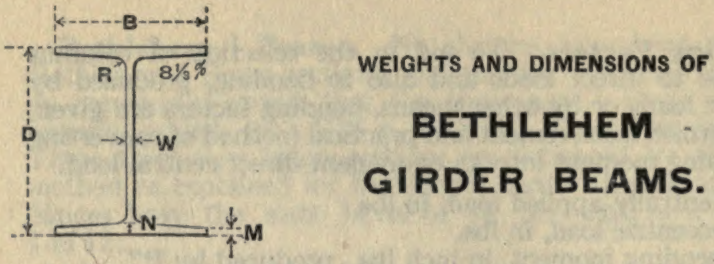

\begin{tabular}{|c|c|c|c|c|c|c|c|}
\hline \multirow{3}{*}{$\begin{array}{l}\text { Section } \\
\text { Number. }\end{array}$} & \multirow{3}{*}{$\begin{array}{c}\text { Weight, } \\
\text { Lbs. } \\
\text { per Foot. }\end{array}$} & \multirow{3}{*}{$\begin{array}{c}\text { Nominal } \\
\text { Depth. } \\
\text { D } \\
\end{array}$} & \multirow{3}{*}{$\begin{array}{c}\text { Flange } \\
\text { Width. } \\
\text { B } \\
\end{array}$} & \multirow{3}{*}{$\begin{array}{c}\text { Web } \\
\text { Thick- } \\
\text { ness. } \\
\text { W } \\
\end{array}$} & \multirow{2}{*}{\multicolumn{2}{|c|}{ Flange Thickness. }} & \multirow{3}{*}{$\begin{array}{c}\text { Radius } \\
\text { of } \\
\text { Fillet. } \\
\text { R } \\
\end{array}$} \\
\hline & & & & & & & \\
\hline & & & & & $\begin{array}{c}\text { At Edge. } \\
\mathbf{M}\end{array}$ & $\begin{array}{c}\text { At Root. } \\
\mathbf{N} \\
\end{array}$ & \\
\hline \multirow{3}{*}{ G30 } & 200.0 & $301 / 8$ & 15.04 & .76 & .950 & 1.545 & .85 \\
\hline & 190.0 & 30 & 15.00 & .72 & .890 & 1.485 & .85 \\
\hline & 181.0 & $297 / 8$ & 14.97 & .69 & .830 & 1.425 & .85 \\
\hline \multirow{2}{*}{ G28 } & 175.0 & $281 / 8$ & 14.29 & .70 & .870 & 1.436 & .80 \\
\hline & 165.0 & 28 & 14.25 & .66 & .810 & 1.376 & .80 \\
\hline \multirow{3}{*}{ G26 } & 160.0 & $261 / 8$ & 13.79 & .67 & .845 & 1.392 & .75 \\
\hline & 151.0 & 26 & 13.75 & .63 & .785 & 1.332 & .75 \\
\hline & 144.0 & $257 / 8$ & 13.73 & .61 & .725 & 1.272 & .75 \\
\hline \multirow{3}{*}{ G24 a } & 149.0 & $241 / 8$ & 13.29 & .65 & .840 & 1.367 & .70 \\
\hline & 141.0 & 24 & 13.25 & .61 & .780 & 1.307 & .70 \\
\hline & 133.0 & $237 / 8$ & 13.22 & .58 & .720 & 1.247 & .70 \\
\hline \multirow{3}{*}{ G24 } & 129.0 & $241 / 8$ & 12.29 & .58 & .770 & 1.258 & .65 \\
\hline & 121.0 & 24 & 12.25 & .54 & .710 & 1.198 & .65 \\
\hline & 114 & $237 / 8$ & 12.22 & .51 & .650 & 1.138 & .65 \\
\hline \multirow{3}{*}{$\mathrm{G} 20 \mathrm{a}$} & 149.0 & $201 / 8$ & 12.78 & .69 & .970 & 1.475 & .75 \\
\hline & 142.0 & 20 & 12.75 & .66 & .910 & 1.415 & .75 \\
\hline & 135.0 & $197 / 8$ & 12.72 & .63 & .850 & 1.355 & .75 \\
\hline \multirow{3}{*}{ G20 } & 120.0 & $201 / 8$ & 12.03 & .59 & .770 & 1.247 & .65 \\
\hline & 113.0 & 20 & 12.00 & .56 & .710 & 1.187 & .65 \\
\hline & 107.0 & $197 / 8$ & 11.98 & .54 & .650 & 1.127 & .65 \\
\hline \multirow{3}{*}{ G18 } & 100.0 & $181 / 8$ & 11.54 & .52 & .670 & 1.129 & .60 \\
\hline & 93.0 & 18 & 11.50 & .48 & .610 & 1.069 & .60 \\
\hline & 87.5 & $177 / 8$ & 11.48 & .46 & .550 & 1.009 & .60 \\
\hline
\end{tabular}


BETHLEHEM STEEL COMPANY.

WEIGHTS AND DIMENSIONS OF

BETHLEHEM

GIRDER BEAMS.

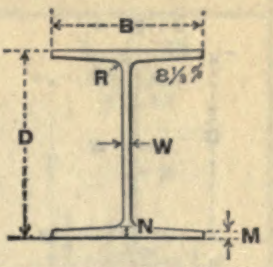

\begin{tabular}{|c|c|c|c|c|c|c|c|}
\hline \multirow{3}{*}{$\begin{array}{l}\text { Section } \\
\text { Number. }\end{array}$} & \multirow{3}{*}{$\begin{array}{l}\text { Weight, } \\
\text { Lbs. } \\
\text { per Foot. }\end{array}$} & \multicolumn{6}{|c|}{ DIMENSIONS, IN INCHES. } \\
\hline & & \multirow{2}{*}{$\begin{array}{c}\text { Nominal } \\
\text { Depth. } \\
\text { D }\end{array}$} & \multirow{2}{*}{$\begin{array}{c}\text { Flange } \\
\text { Width. } \\
\text { B }\end{array}$} & \multirow{2}{*}{$\begin{array}{c}\text { Web } \\
\text { Thick- } \\
\text { ness. } \\
\text { W }\end{array}$} & \multicolumn{2}{|c|}{ Flange Thickness. } & \multirow{2}{*}{$\begin{array}{c}\begin{array}{c}\text { Radius } \\
\text { of } \\
\text { Fillet. } \\
\text { R }\end{array} \\
\end{array}$} \\
\hline & & & & & $\begin{array}{c}\text { At Edge. } \\
\mathbf{M} \\
\end{array}$ & $\begin{array}{c}\text { At Root. } \\
\mathbf{N} \\
\end{array}$ & \\
\hline \multirow{3}{*}{$\mathrm{G} 15 \mathrm{~b}$} & 147.0 & $151 / 8$ & 11.78 & .83 & 1.150 & 1.606 & .90 \\
\hline & 141.0 & 15 & 11.75 & .80 & 1.090 & 1.546 & .90 \\
\hline & 135.0 & $147 / 8$ & 11.72 & .77 & 1.030 & 1.486 & .90 \\
\hline \multirow{3}{*}{ G15 a } & 111.0 & $151 / 8$ & 11.29 & .64 & .845 & 1.289 & .70 \\
\hline & 105.0 & 15 & 11.25 & .60 & .785 & 1.229 & .70 \\
\hline & 99.0 & $147 / 8$ & 11.22 & .57 & .725 & 1.169 & .70 \\
\hline \multirow{3}{*}{ G15 } & 80.5 & $151 / 8$ & 10.79 & .48 & .570 & 1.000 & .55 \\
\hline & 74.0 & 15 & 10.75 & .44 & .510 & .940 & .55 \\
\hline & 69.0 & $147 / 8$ & 10.73 & .42 & .450 & .880 & .55 \\
\hline \multirow{3}{*}{ G12 a } & 76.5 & $121 / 8$ & 10.29 & .51 & .620 & 1.027 & .55 \\
\hline & 70.5 & 12 & 10.25 & .47 & .560 & .967 & .55 \\
\hline & 66.0 & $117 / 8$ & 10.23 & .45 & .500 & .907. & .55 \\
\hline \multirow{3}{*}{ G12 } & 61.0 & $121 / 8$ & 10.03 & .41 & .465 & .866 & .45 \\
\hline & 55.5 & 12 & 10.00 & .38 & .405 & .806 & .45 \\
\hline & 51.5 & $11 \frac{29}{32}$ & 9.98 & .36 & .360 & .761 & .45 \\
\hline \multirow{3}{*}{ G10 } & 50.0 & $101 / 8$ & 9.04 & .36 & .445 & .807 & .40 \\
\hline & 44.5 & 10 & 9.00 & .32 & .385 & .747 & .40 \\
\hline & 41.5 & $9 \frac{29}{3 \frac{2}{2}}$ & 8.99 & .31 & .340 & .702 & .40 \\
\hline \multirow{3}{*}{ G9 } & 43.5 & $91 / 8$ & 8.54 & .35 & .405 & .746 & .40 \\
\hline & 38.5 & 9 & 8.50 & .31 & .345 & .686 & .40 \\
\hline & 36.0 & $8 \frac{15}{16}$ & 8.48 & .29 & .315 & .656 & .40 \\
\hline \multirow{3}{*}{ G8 } & 37.0 & $81 / 8$ & 8.03 & .33 & .365 & .686 & .40 \\
\hline & 33.0 & 8 & 8.00 & .30 & .305 & .626 & .40 \\
\hline & 31.0 & $7 \frac{15}{16}$ & 7.99 & .29 & .275 & .596 & .40 \\
\hline
\end{tabular}


BETHLEHEM STEEL COMPANY.

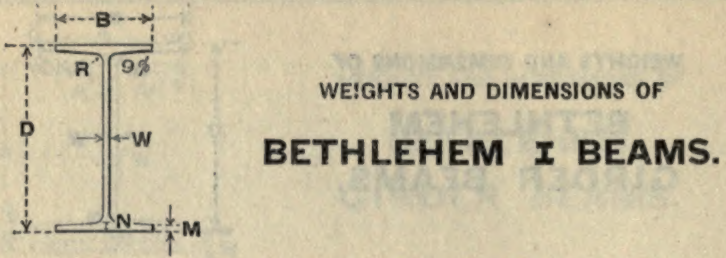

\begin{tabular}{|c|c|c|c|c|c|c|c|}
\hline \multirow{3}{*}{$\begin{array}{l}\text { Section } \\
\text { Number. }\end{array}$} & \multirow{3}{*}{$\begin{array}{c}\text { Weight, } \\
\text { Lbs. } \\
\text { per Foot. }\end{array}$} & \multicolumn{6}{|c|}{ DIMENSIONS, IN INCHES. } \\
\hline & & \multirow[b]{2}{*}{$\begin{array}{c}\text { Depth. } \\
\text { D }\end{array}$} & \multirow[b]{2}{*}{$\begin{array}{c}\text { Flange } \\
\text { Width. } \\
\text { B }\end{array}$} & \multirow{2}{*}{$\begin{array}{c}\text { Web } \\
\text { Thick- } \\
\text { ness. } \\
\text { W }\end{array}$} & \multicolumn{2}{|c|}{ Flange Thickness. } & \multirow{2}{*}{$\begin{array}{c}\text { Radius } \\
\text { of } \\
\text { Fillet. } \\
\text { R }\end{array}$} \\
\hline & & & & & $\begin{array}{c}\text { At Edge. } \\
\mathbf{M}\end{array}$ & $\begin{array}{c}\text { At Root. } \\
\mathbf{N}\end{array}$ & \\
\hline $\mathrm{B} 30$ & 121.0 & 30 & 10.500 & .540 & .735 & 1.183 & .65 \\
\hline B28 & 106.0 & 28 & 10.000 & .500 & .675 & 1.102 & .60 \\
\hline B26 & 91.0 & 26 & 9.500 & .460 & .600 & 1.007 & .55 \\
\hline B24 b* & $\begin{array}{r}104.5 \\
99.5 \\
95.5\end{array}$ & $\begin{array}{l}24 \frac{3}{32} \\
24 \\
23 \frac{29}{32}\end{array}$ & $\begin{array}{l}9.775 \\
9.750 \\
9.730\end{array}$ & $\begin{array}{l}.550 \\
.525 \\
.505\end{array}$ & $\begin{array}{l}.750 \\
.705 \\
.660\end{array}$ & $\begin{array}{l}1.134 \\
1.089 \\
1.044\end{array}$ & $\begin{array}{l}.60 \\
.60 \\
.60\end{array}$ \\
\hline B24 a & 84.5 & 24 & 9.250 & .460 & .585 & .980 & .55 \\
\hline B24 & $\begin{array}{l}83.0 \\
73.5\end{array}$ & $\begin{array}{l}24 \\
24\end{array}$ & $\begin{array}{l}9.130 \\
\mathbf{9 . 0 0 0}\end{array}$ & $\begin{array}{l}.520 \\
.390\end{array}$ & $\begin{array}{l}.510 \\
.510\end{array}$ & $\begin{array}{l}.897 \\
.897\end{array}$ & $\begin{array}{l}.50 \\
.50\end{array}$ \\
\hline B22* & $\begin{array}{l}71.5 \\
68.5 \\
\mathbf{6 5 . 5}\end{array}$ & $\begin{array}{l}221 / 8 \\
22 \frac{1}{16} \\
22\end{array}$ & $\begin{array}{l}8.535 \\
8.520 \\
\mathbf{8 . 5 0 0}\end{array}$ & $\begin{array}{l}.420 \\
.405 \\
.385\end{array}$ & $\begin{array}{l}.545 \\
.515 \\
.485\end{array}$ & $\begin{array}{l}.883 \\
.853 \\
.823\end{array}$ & $\begin{array}{l}.50 \\
.50 \\
.50\end{array}$ \\
\hline B20 a & $\begin{array}{l}82.0 \\
73.0\end{array}$ & $\begin{array}{l}20 \\
20\end{array}$ & $\begin{array}{l}8.890 \\
8.750\end{array}$ & $\begin{array}{l}.570 \\
.430\end{array}$ & $\begin{array}{l}.580 \\
.580\end{array}$ & $\begin{array}{l}.955 \\
.955\end{array}$ & $\begin{array}{l}.55 \\
.55\end{array}$ \\
\hline $\mathrm{B} 20$ & $\begin{array}{l}69.0 \\
64.5 \\
59.5\end{array}$ & $\begin{array}{l}20 \\
20 \\
20\end{array}$ & $\begin{array}{l}8.145 \\
8.075 \\
8.000\end{array}$ & $\begin{array}{l}.520 \\
.450 \\
.375\end{array}$ & $\begin{array}{l}.475 \\
.475 \\
.475\end{array}$ & $\begin{array}{l}.818 \\
.818 \\
.818\end{array}$ & $\begin{array}{l}.45 \\
.45 \\
.45\end{array}$ \\
\hline
\end{tabular}

Note: All Bethlehem I Beams have a slope of flange of $9 \%$ except those marked $*$ which have a slope of $8 \frac{1}{3} \%$. 
BETHLEHEM STEEL. COMPANY.

\section{WEIGHTS AND DIMENSIONS OF. BETHLEHEM I BEAMS.}

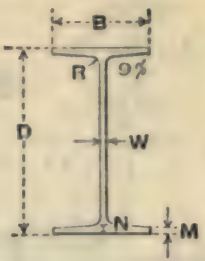

\begin{tabular}{|c|c|c|c|c|c|c|c|}
\hline \multirow{3}{*}{$\begin{array}{l}\text { Section } \\
\text { Number. }\end{array}$} & \multirow{3}{*}{$\begin{array}{l}\text { Weight, } \\
\text { Lbs. } \\
\text { per Foot. }\end{array}$} & \multicolumn{6}{|c|}{ DIMENSIONS, IN INCHES. } \\
\hline & & \multirow[b]{2}{*}{$\begin{array}{c}\text { Depth. } \\
\text { D }\end{array}$} & \multirow[b]{2}{*}{$\begin{array}{c}\text { Flange } \\
\text { Width. } \\
\text { B }\end{array}$} & \multirow{2}{*}{$\begin{array}{c}\text { Web } \\
\text { Thick- } \\
\text { ness. } \\
\text { W }\end{array}$} & \multicolumn{2}{|c|}{ Flange Thickness. } & \multirow{2}{*}{$\begin{array}{c}\begin{array}{c}\text { Radius } \\
\text { of } \\
\text { Fillet. } \\
\text { R }\end{array} \\
\end{array}$} \\
\hline & & & & & $\begin{array}{c}\text { At Edge. } \\
\text { M }\end{array}$ & $\begin{array}{l}\text { At Root. } \\
\qquad \mathbf{N}\end{array}$ & \\
\hline $\mathrm{B} 18 \mathrm{a}^{*}$ & $\begin{array}{l}74.0 \\
69.0 \\
64.5\end{array}$ & $\begin{array}{l}181 / 8 \\
18 \\
177 / 8\end{array}$ & $\begin{array}{l}8.770 \\
8.750 \\
8.730\end{array}$ & $\begin{array}{l}.440 \\
.420 \\
.400\end{array}$ & $\begin{array}{l}.645 \\
.585 \\
.525\end{array}$ & $\begin{array}{l}.992 \\
.932 \\
.872\end{array}$ & $\begin{array}{l}.50 \\
.50 \\
.50\end{array}$ \\
\hline B18 & $\begin{array}{l}59.0 \\
54.5 \\
52.0 \\
49.0\end{array}$ & $\begin{array}{l}18 \\
18 \\
18 \\
18\end{array}$ & $\begin{array}{l}7.675 \\
7.590 \\
7.555 \\
\mathbf{7 . 5 0 0}\end{array}$ & $\begin{array}{l}.495 \\
.410 \\
.375 \\
.320\end{array}$ & $\begin{array}{l}.430 \\
.430 \\
.430 \\
.430\end{array}$ & $\begin{array}{l}.753 \\
.753 \\
.753 \\
.753\end{array}$ & $\begin{array}{l}.40 \\
.40 \\
.40 \\
.40\end{array}$ \\
\hline $\mathrm{B} 15 \mathrm{~b}$ & 71.5 & 15 & 7.500 & .520 & .785 & 1.099 & .60 \\
\hline $\mathrm{B} 15 \mathrm{a}$ & $\begin{array}{l}64.0 \\
54.5\end{array}$ & $\begin{array}{l}15 \\
15\end{array}$ & $\begin{array}{l}7.195 \\
7.000\end{array}$ & $\begin{array}{l}.605 \\
.410\end{array}$ & $\begin{array}{l}.590 \\
.590\end{array}$ & $\begin{array}{l}.887 \\
.887\end{array}$ & $\begin{array}{l}.50 \\
.50\end{array}$ \\
\hline B15 & $\begin{array}{l}46.0 \\
41.0 \\
38.5\end{array}$ & $\begin{array}{l}15 \\
15 \\
15\end{array}$ & $\begin{array}{l}6.810 \\
6.710 \\
6.660\end{array}$ & $\begin{array}{l}.440 \\
.340 \\
.290\end{array}$ & $\begin{array}{l}.400 \\
.400 \\
.400\end{array}$ & $\begin{array}{l}.686 \\
.686 \\
.686\end{array}$ & $\begin{array}{l}.40 \\
.40 \\
.40\end{array}$ \\
\hline $\mathrm{B} 12 \mathrm{a}$ & 36.5 & 12 & 6.300 & .310 & .440 & .710 & .40 \\
\hline B12 & $\begin{array}{l}32.0 \\
28.5\end{array}$ & $\begin{array}{l}12 \\
12\end{array}$ & $\begin{array}{l}6.205 \\
6.120\end{array}$ & $\begin{array}{l}.335 \\
.250\end{array}$ & $\begin{array}{l}.330 \\
.330\end{array}$ & $\begin{array}{l}.594 \\
.594\end{array}$ & $\begin{array}{l}.35 \\
.35\end{array}$ \\
\hline $\mathrm{B} 10$ & $\begin{array}{l}28.5 \\
23.5\end{array}$ & $\begin{array}{l}10 \\
10\end{array}$ & $\begin{array}{l}5.990 \\
\mathbf{5 . 8 5 0}\end{array}$ & $\begin{array}{l}.390 \\
.250\end{array}$ & $\begin{array}{l}.270 \\
.270\end{array}$ & $\begin{array}{l}.522 \\
.522\end{array}$ & $\begin{array}{l}.30 \\
.30\end{array}$ \\
\hline B!) & $\begin{array}{l}24.0 \\
20.5\end{array}$ & 9 & $\begin{array}{l}5.555 \\
\mathbf{5 . 4 4 0}\end{array}$ & $\begin{array}{l}.365 \\
.250\end{array}$ & $\begin{array}{l}.245 \\
.245\end{array}$ & $\begin{array}{l}.479 \\
.479\end{array}$ & $\begin{array}{l}.30 \\
.30\end{array}$ \\
\hline B 88 & $\begin{array}{l}19.5 \\
17.5\end{array}$ & $\begin{array}{l}8 \\
8\end{array}$ & $\begin{array}{l}5.325 \\
5.250\end{array}$ & $\begin{array}{l}.325 \\
.250\end{array}$ & $\begin{array}{l}.205 \\
.205\end{array}$ & $\begin{array}{l}.430 \\
.430\end{array}$ & $\begin{array}{l}.30 \\
.30\end{array}$ \\
\hline
\end{tabular}

Notc: All Bethlchem I Beams have a slope of tlange of $9 \%$ except those marked * which have a slope of $8 \frac{1}{3} \%$. 
BETHLEHEM STEEL COMPANY.

\begin{tabular}{|c|c|c|c|c|c|c|c|c|}
\hline \multicolumn{2}{|c|}{$Y_{i}^{\prime}$} & \multirow{2}{*}{\multicolumn{7}{|c|}{ PROPERTIES OF }} \\
\hline$x$ & & & & & & & & \\
\hline$=$ & Y & \multicolumn{3}{|c|}{ BETHLEHEM } & \multicolumn{2}{|c|}{ GIRDER } & \multicolumn{2}{|c|}{ BEAMS. } \\
\hline \multirow[b]{2}{*}{$\begin{array}{l}\text { Section } \\
\text { Number. }\end{array}$} & \multirow[b]{2}{*}{$\begin{array}{l}\text { Nominal } \\
\text { Depth } \\
\text { of } \\
\text { Beam, } \\
\text { Inches. }\end{array}$} & \multirow[b]{2}{*}{$\begin{array}{c}\text { Weight, } \\
\text { Lbs. } \\
\text { per } \\
\text { Foot. }\end{array}$} & \multirow[b]{2}{*}{$\begin{array}{c}\text { Area } \\
\text { of } \\
\text { Section, } \\
\text { Square } \\
\text { Inches. }\end{array}$} & \multirow[b]{2}{*}{$\begin{array}{l}\text { Thick- } \\
\text { ness } \\
\text { of } \\
\text { Web, } \\
\text { Inches. }\end{array}$} & \multirow[b]{2}{*}{$\begin{array}{l}\text { Width } \\
\text { of } \\
\text { Flange, } \\
\text { Inches. }\end{array}$} & \multicolumn{3}{|c|}{ AXIS $X X$. } \\
\hline & & & & & & $\begin{array}{c}\text { Moment } \\
\text { of } \\
\text { Inertia. } \\
\text { I }\end{array}$ & $\begin{array}{c}\text { Radius } \\
\text { of } \\
\text { Gyra- } \\
\text { tion. } \\
\\
\mathbf{r}\end{array}$ & $\begin{array}{c}\text { Section } \\
\text { Modulus } \\
\text { s }\end{array}$ \\
\hline G30 & $\begin{array}{l}301 / 8 \\
30 \\
297 / 8\end{array}$ & $\begin{array}{l}200.0 \\
190.0 \\
181.0\end{array}$ & $\begin{array}{l}58.52 \\
55.52 \\
52.82\end{array}$ & $\begin{array}{l}.76 \\
.72 \\
.69\end{array}$ & $\begin{array}{l}15.04 \\
15.00 \\
14.97\end{array}$ & $\begin{array}{l}9148.8 \\
8651.1 \\
8181.0\end{array}$ & $\begin{array}{l}12.50 \\
12.48 \\
12.45\end{array}$ & $\begin{array}{l}607.5 \\
576.7 \\
547.6\end{array}$ \\
\hline G28 & $\begin{array}{l}281 / 8 \\
28\end{array}$ & $\begin{array}{l}175.0 \\
165.0\end{array}$ & $\begin{array}{l}51.02 \\
48.19\end{array}$ & $\begin{array}{l}.70 \\
.66\end{array}$ & $\begin{array}{l}14.29 \\
14.25\end{array}$ & $\begin{array}{l}6988.7 \\
6577.9\end{array}$ & $\begin{array}{l}11.70 \\
11.68\end{array}$ & $\begin{array}{l}497.1 \\
469.9\end{array}$ \\
\hline G26 & $\begin{array}{l}261 / 8 \\
26 \\
257 / 8\end{array}$ & $\begin{array}{l}160.0 \\
151.0 \\
144.0\end{array}$ & $\begin{array}{l}46.85 \\
44.16 \\
41.99\end{array}$ & $\begin{array}{l}.67 \\
.63 \\
.61\end{array}$ & $\begin{array}{l}13.79 \\
13.75 \\
13.73\end{array}$ & $\begin{array}{l}5576.6 \\
5237.1 \\
4930.6\end{array}$ & $\begin{array}{l}10.91 \\
10.89 \\
10.84\end{array}$ & $\begin{array}{l}427.0 \\
402.9 \\
381.0\end{array}$ \\
\hline G24 a & $\begin{array}{l}241 / 8 \\
24 \\
237 / 8\end{array}$ & $\begin{array}{l}149.0 \\
141.0 \\
133.0\end{array}$ & $\begin{array}{l}43.57 \\
41.02 \\
38.71\end{array}$ & $\begin{array}{l}.65 \\
.61 \\
.58\end{array}$ & $\begin{array}{l}13.29 \\
13.25 \\
13.22\end{array}$ & $\begin{array}{l}4451.1 \\
4174.2 \\
3912.4\end{array}$ & $\begin{array}{l}10.11 \\
10.09 \\
10.05\end{array}$ & $\begin{array}{l}369.1 \\
347.9 \\
327.7\end{array}$ \\
\hline G24 & $\begin{array}{l}241 / 8 \\
24 \\
237 / 8\end{array}$ & \begin{tabular}{|l|}
129.0 \\
121.0 \\
114.0
\end{tabular} & \begin{tabular}{|l|}
37.74 \\
35.30 \\
33.12
\end{tabular} & $\begin{array}{l}.58 \\
.54 \\
.51\end{array}$ & $\begin{array}{l}12.29 \\
12.25 \\
12.22\end{array}$ & $\begin{array}{l}3844.8 \\
3585.3 \\
3340.6\end{array}$ & $\begin{array}{l}10.09 \\
10.08 \\
10.04\end{array}$ & $\begin{array}{l}318.8 \\
298.8 \\
279.8\end{array}$ \\
\hline G20 a & $\begin{array}{l}201 / 8 \\
20 \\
197 / 8\end{array}$ & $\begin{array}{l}149.0 \\
142.0 \\
135.0\end{array}$ & \begin{tabular}{|l|}
43.44 \\
41.31 \\
39.18
\end{tabular} & $\begin{array}{l}.69 \\
.66 \\
.63\end{array}$ & $\begin{array}{l}12.78 \\
12.75 \\
12.72\end{array}$ & $\begin{array}{l}3106.6 \\
2932.3 \\
2760.6\end{array}$ & $\begin{array}{l}8.46 \\
8.43 \\
8.39\end{array}$ & $\begin{array}{l}308.8 \\
293.2 \\
277.7\end{array}$ \\
\hline G20 & $\begin{array}{l}201 / 8 \\
20 \\
197 / 8\end{array}$ & $\begin{array}{l}120.0 \\
113.0 \\
107.0\end{array}$ & $\begin{array}{l}34.95 \\
32.90 \\
31.06\end{array}$ & $\begin{array}{l}.59 \\
.56 \\
.54\end{array}$ & $\begin{array}{l}12.03 \\
12.00 \\
11.98\end{array}$ & $\begin{array}{l}2505.5 \\
2340.2 \\
2184.0\end{array}$ & $\begin{array}{l}8.47 \\
8.43 \\
8.39\end{array}$ & $\begin{array}{l}249.1 \\
234.0 \\
219.7\end{array}$ \\
\hline G18 & $\begin{array}{l}181 / 8 \\
18 \\
177 / 8\end{array}$ & $\begin{array}{r}100.0 \\
93.0 \\
87.5\end{array}$ & \begin{tabular}{|l|}
29.25 \\
27.14 \\
25.40
\end{tabular} & $\begin{array}{l}.52 \\
.48 \\
.46\end{array}$ & $\begin{array}{l}11.54 \\
11.50 \\
11.48\end{array}$ & $\begin{array}{l}1725.7 \\
1593.4 \\
1472.8\end{array}$ & $\begin{array}{l}7.68 \\
7.66 \\
7.61\end{array}$ & $\begin{array}{l}190.5 \\
177.0 \\
164.7 \\
\end{array}$ \\
\hline $\begin{array}{l}\mathbf{W}=\mathrm{S} \\
\mathbf{L}=\mathrm{S} \\
\mathbf{M}=\mathrm{N} \\
\mathbf{f}=\mathrm{A} \\
\mathbf{S}=\mathrm{S}\end{array}$ & $\mathrm{M}$ & St & 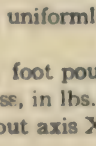 & $\mathrm{er} 8$ & in & & & \\
\hline
\end{tabular}


BETHLEHEM STEEL COMPANY.

PROPERTIES OF

\section{BETHLEHEM GIRDER BEAMS.}

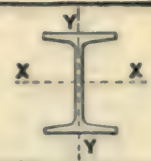

\section{COEFFICIENTS OF STRENGTH.}

For Fiber
Stress of
$16,(000)$ Lbs.
per Sq. In.
UsualBuild-
ingCodeRe-
quirement.
C

$6,480,000$

$6,152,000$

$5,841,000$

$5,302,000$

$5,012,000$

$4,555,000$

$4,297,000$

$4,064,000$

$3,937,000$

$3,710,000$

$3,495,000$

$3,401,000$

$3,187,000$

$2,984,000$

$2,550,000$

$3,826,000114,800$

For Fiber

Siress of

For Fiber

12, 18,000 Lbs.

For Moving For Quies-

Loads.

$\frac{\text { C }^{\prime}}{4,860,000}$ cent Loads.

$4,614,000$

C" $^{\prime \prime}$

193,200

$7,290,000193,200$

$4,381,000$

$6,571,000163,700$

$3,976,000$

$5,965,000164,800$

$3,759,000$

$5,638,000149,100$

\section{$3,416,000$}

$5,124,000149,500$

$3,223,000$

$4,834,000134,900$

$3,048,000$

$4,572,000127,300$

$2,953,000$

$4,429,000138,200$

$2,783,000$

$4,174,000124,600$

$2,621,000$

$3,932,000114,400$

$2,390,000$

$3,585,000101,400$

$2,238,000$

$3,357,000$

91,400

$3,294,000$

$2,470,000$

$3,706,000138,100$

$3,128,000$

$2,962,000$

$2,346,000$

$3,519,000129,200$

$3,333,000120,500$

$2,657,000$

$2,222,000$

$2,989,000109,800$

$1,992,000$

$2,808,000101,000$

$2,496,000$

$1,872,000$

$2,637,00095,100$

$2,344,000$

$1,758,000$

$2,286,00086,300$

$2,032,000$

$1,524,000$

$2,125,000$

76,000

$1,888,000 \quad 1,416,000$

$\begin{array}{lllll}1,757,000 & 1,318,000 & 1,977,000 & 70,700\end{array}$
AXIS $Y Y$.

496.2

462.8

432.8

402.7

375.0

383.3

356.4

330.7

278.2

256.9

236.7

384.5

360.9

337.6

260.1

240.8

222.3

202.6

185.1

168.9
Radiu
of
Gyra
tion.
$r^{\prime}$

3.28

3.26

3.23

3.12

3.10

3.04

3.02

2.99

2.97

2.95

2.92

2.72

2.70

2.67

2.97

2.96

2.94

2.73

2.71

2.68

2.63

2.61

2.58
Section

Section Num
Modulus.

$\mathbf{S}^{\prime}$

83.6

78.6

73.7

69.4

65.0

62.8

58.6

54.6

57.7

53.8

50.0

45.3

41.9

38.7

60.2

56.6

53.1

43.2

40.1

37.1

35.1

32.2

29.4

G30

G28

G 36

G24 a

G24

G20a
$\mathbf{C}, \mathbf{C}^{\prime}$, and $\mathbf{C}^{\prime \prime}=$ Coefficients given in the table.

$\mathbf{W}=\frac{C}{\mathbf{L}}$, or $\frac{C^{\prime}}{\mathbf{L}^{\prime}}$, or $\frac{C^{\prime \prime}}{\mathbf{L}} ; \mathbf{M}=\frac{C^{\prime}}{8}$ or $\frac{C^{\prime}}{8}$, or $\frac{C^{\prime \prime}}{8}$

$C$, or $C^{\prime}$, or $C^{\prime \prime}=W L-8 M=$ i 18 
BETHLEHEM STEEL COMPANY.

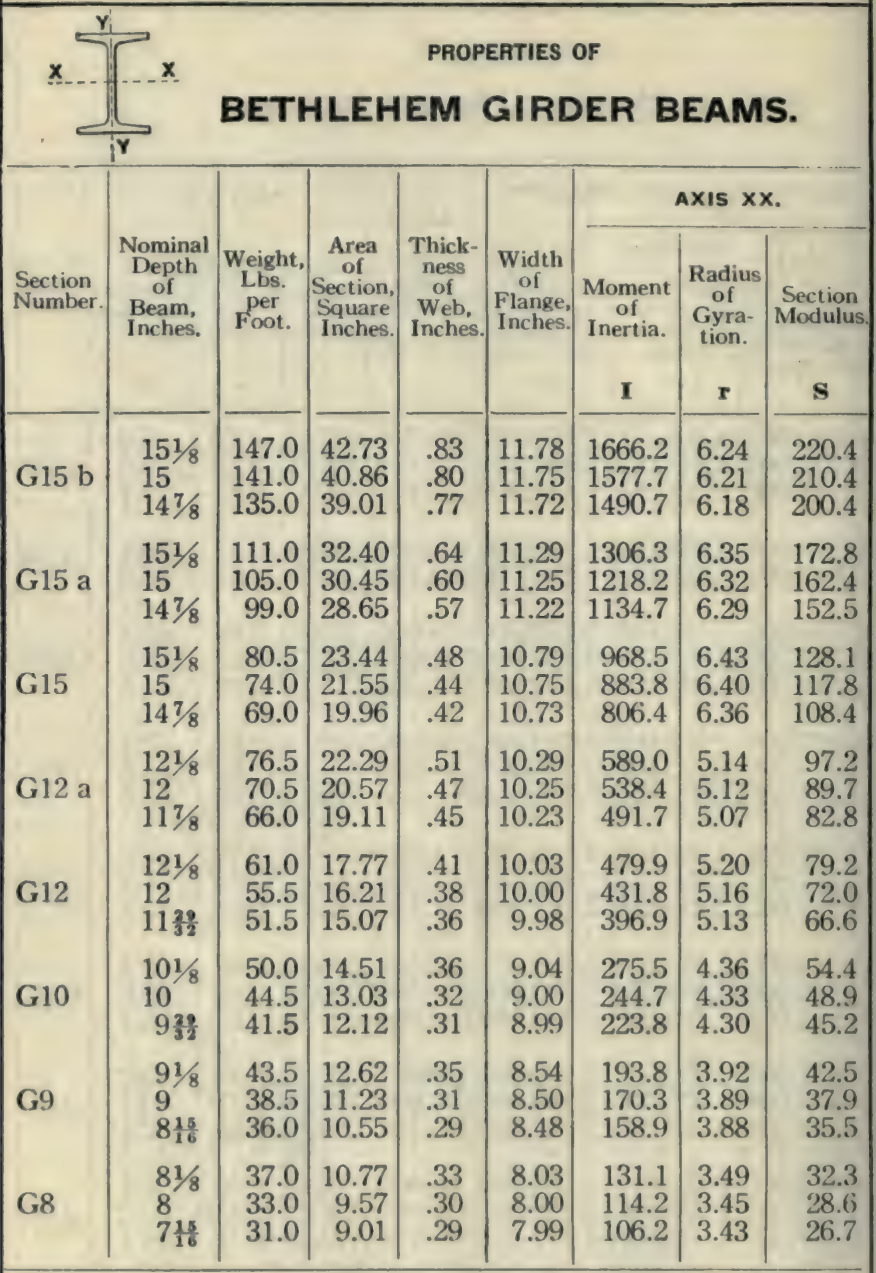

$\mathbf{W}=$-Safe load in pounds, uniformly distributed, including weight of beam.

$\mathbf{L}=$ Span, in feet.

$\mathbf{M}=$ Moment of forces, in foot pounds.

f - Allowable Fiber Stress, in lbs. per square inch.

$\mathbf{S}=$ Section Modulus about axis XX. 
PROPERTIES OF BETHLEHEM GIRDER BEAMS.

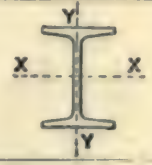

\section{COEFFICIENTS OF STRENGTH.}

For Fiber | For Fiber | For Fiber Stress of Furess of Stress of 16,(0) Lbs. 12,000 Lbs. 18,000 Lbs. UsualBuild per Sq. In. per Sq. In. ungCudeke-For Moving For Quiesyuirement. Loads, cent Loads.

C

$$
\mathbf{C}^{\prime}
$$

$2,351,0001,763,0002,645,000125,500$ $2,244,0001,683,0002,524,000120,000$ $2,137,0001.603,0002,404,000 \quad 114,600$

$1,843,0001,382,0002,074,000$ $1,733,0001,299,0001,949,000$ $1,627,0001,220,0001,830,000$

$1,367,0001,025,0001,537,000$ $1,257,000 \quad 942,8001,414,000$ $1,156,000 \quad 867,1001,301,000$

\section{$1,037,000$ $777,6001,166,000$} 957,100 $717,8001,077,000$ 883,000

844,700 767,700 710,800

580,800

522,100 481,900

453,400 403,800 662,300 993,400

96,800 90,000 84,800

69,700 61,100 56,600 61,800 56,400 53,500 633,500 575,800 533,100

950,300 863,600

49,100 43,800 799,700

435,600 391,500 361,400

653,300 587,300 542,100 40,300

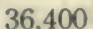

31,100

29,500

340,000 302,800 510,000 454,300 379,200 284,400

344,500 258,400 304,500 228,400 285,200
426,600

387,600 342,600 320,900
31,900

27,900

25,300

26,800

24,000 23,000

\begin{tabular}{|c|c|c|c|}
\hline \multicolumn{3}{|c|}{ AXIS YY. } & \multirow[b]{2}{*}{$\begin{array}{l}\text { Sertion } \\
\text { Num- } \\
\text { ber. }\end{array}$} \\
\hline $\begin{array}{c}\text { Mo- } \\
\text { ment } \\
\text { of } \\
\text { Inertia. }\end{array}$ & $\begin{array}{c}\text { Radius } \\
\text { of } \\
\text { Gyra- } \\
\text { tion. } \\
\mathbf{r}^{\prime}\end{array}$ & $\begin{array}{c}\text { Section } \\
\text { Modulus. } \\
\text { s' }\end{array}$ & \\
\hline $\begin{array}{l}347.3 \\
328.3 \\
309.5\end{array}$ & $\begin{array}{l}2.85 \\
2.83 \\
2.82\end{array}$ & $\begin{array}{l}59.0 \\
55.9 \\
52.8\end{array}$ & G15b \\
\hline $\begin{array}{l}231.2 \\
214.3 \\
198.4\end{array}$ & $\begin{array}{l}2.67 \\
2.65 \\
2.63\end{array}$ & $\begin{array}{l}41.0 \\
38.1 \\
35.4\end{array}$ & G15a \\
\hline $\begin{array}{l}143.0 \\
128.9 \\
115.8\end{array}$ & $\begin{array}{l}2.47 \\
2.45 \\
2.41\end{array}$ & $\begin{array}{l}26.5 \\
24.0 \\
21.6\end{array}$ & G15 \\
\hline $\begin{array}{l}132.1 \\
119.7 \\
108.3\end{array}$ & $\begin{array}{l}2.43 \\
2.41 \\
2.38\end{array}$ & $\begin{array}{l}25.7 \\
23.4 \\
21.2\end{array}$ & G12a \\
\hline $\begin{array}{l}95.8 \\
84.9 \\
76.9\end{array}$ & $\begin{array}{l}2.32 \\
2.29 \\
2.26\end{array}$ & $\begin{array}{l}19.1 \\
17.0 \\
15.4\end{array}$ & G12 \\
\hline $\begin{array}{l}66.4 \\
58.2 \\
52.6\end{array}$ & $\begin{array}{l}2.14 \\
2.11 \\
2.08\end{array}$ & $\begin{array}{l}14.7 \\
12.9 \\
11.7\end{array}$ & G10 \\
\hline $\begin{array}{l}51.3 \\
44.4 \\
41.0\end{array}$ & $\begin{array}{l}2.02 \\
1.99 \\
1.97\end{array}$ & $\begin{array}{c}12.0 \\
10.4 \\
9.67\end{array}$ & G9 \\
\hline $\begin{array}{l}38.7 \\
33.2 \\
30.5\end{array}$ & $\begin{array}{l}1.90 \\
1.86 \\
1.84\end{array}$ & $\begin{array}{l}9.65 \\
8.29 \\
7.63\end{array}$ & G8 \\
\hline
\end{tabular}

AXIS YY.

C. $\mathrm{C}^{\prime}$, and $\mathrm{C}^{\prime \prime}=$ Coefficients given in the table.

$\mathbf{W}=\frac{\mathbf{C}}{\mathbf{I}}$, or $\frac{\mathbf{C}^{\prime}}{\mathbf{L}}$, or $\frac{\mathbf{C}^{\prime \prime}}{\mathbf{L}} ; \mathbf{M}=\frac{\mathbf{C}}{\mathbf{8}}$, or $\frac{\mathbf{C}^{\prime}}{8}$, or $\frac{\mathbf{C}^{\prime \prime}}{8}$

$C$, or $C^{\prime}$, or $C^{\prime \prime \prime}-W L=8 M=\frac{2}{2}$ P $S$ 
BETHLEHEM STEEL COMPANY.

\begin{tabular}{|c|c|c|c|c|c|c|c|c|}
\hline 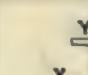 & & & & PROP & IES of & & & \\
\hline & & BE & THL & HER & $4 x$ & BEAM & ns. & \\
\hline & & & & & & & $A x$ & \\
\hline $\begin{array}{l}\text { Section } \\
\text { Number }\end{array}$ & $\begin{array}{l}\text { Depth } \\
\text { of } \\
\text { Beam, } \\
\text { Inches. }\end{array}$ & $\begin{array}{l}\text { Weight. } \\
\text { L.bs. } \\
\text { per } \\
\text { foot. }\end{array}$ & $\begin{array}{c}\text { Area } \\
\text { of } \\
\text { Section } \\
\text { square } \\
\text { Inches }\end{array}$ & $\begin{array}{l}\text { Thick- } \\
\text { ness } \\
\text { of } \\
\text { Web, } \\
\text { Inches. }\end{array}$ & $\begin{array}{l}\text { Width } \\
\text { of } \\
\text { olange. } \\
\text { Inches. }\end{array}$ & $\begin{array}{c}\text { Moment } \\
\text { of } \\
\text { Inertia. } \\
\text { I }\end{array}$ & $\begin{array}{c}\text { Radius } \\
\text { of } \\
\text { Gyra- } \\
\text { tion. } \\
\mathbf{r}\end{array}$ & $\begin{array}{c}\text { Section } \\
\text { Modulus } \\
\text { s }\end{array}$ \\
\hline B30 & 30 & 121.0 & 35.30 & .540 & 10.500 & 5239.6 & 12.18 & 349.3 \\
\hline B28 & 28 & 106.0 & 30.88 & .500 & 10.000 & 4014.1 & 11.40 & 286.7 \\
\hline B26 & 26 & 91.0 & 26.49 & .460 & .500 & 2977.2 & 10.60 & 229.0 \\
\hline B24 b & $\begin{array}{l}24 \frac{3}{32} \\
24 \\
23 \frac{39}{32}\end{array}$ & $\begin{array}{r}104.5 \\
99.5 \\
95.5\end{array}$ & $\begin{array}{l}30.63 \\
29.15 \\
27.79\end{array}$ & $\begin{array}{l}.550 \\
.525 \\
.505\end{array}$ & $\begin{array}{l}9.775 \\
9.750 \\
9.730\end{array}$ & $\begin{array}{l}2967.7 \\
2811.7 \\
2663.1\end{array}$ & $\begin{array}{l}9.84 \\
9.82 \\
9.79\end{array}$ & $\begin{array}{l}246.4 \\
234.3 \\
222.8\end{array}$ \\
\hline B24 a & 24 & 84.5 & 24.80 & .460 & 9.250 & 2381.9 & 9.80 & 198.5 \\
\hline B24 & $\begin{array}{l}24 \\
24\end{array}$ & \begin{tabular}{|l|}
83.0 \\
73.5
\end{tabular} & $\begin{array}{l}24.59 \\
21.47\end{array}$ & $\begin{array}{l}.520 \\
.390\end{array}$ & $\begin{array}{l}9.130 \\
9.000\end{array}$ & $\left\{\begin{array}{l}2240.9 \\
2091.0\end{array}\right.$ & $\begin{array}{l}9.55 \\
9.87\end{array}$ & $\begin{array}{l}186.7 \\
174.3\end{array}$ \\
\hline B22 & $\begin{array}{l}221 / 8 \\
22 \frac{1}{16} \\
22\end{array}$ & \begin{tabular}{|l|}
71.5 \\
68.5 \\
65.5
\end{tabular} & \begin{tabular}{|l|}
20.88 \\
20.04 \\
19.08
\end{tabular} & $\begin{array}{l}.420 \\
.405 \\
.385\end{array}$ & $\begin{array}{l}8.535 \\
8.520 \\
8.500\end{array}$ & $\begin{array}{l}1705.2 \\
1629.3 \\
1549.5\end{array}$ & $\begin{array}{l}9.04 \\
9.02 \\
9.01\end{array}$ & $\begin{array}{l}154.2 \\
147.7 \\
140.9\end{array}$ \\
\hline $\mathrm{B} 20 \mathrm{a}$ & $\begin{array}{l}20 \\
20\end{array}$ & \begin{tabular}{|l|}
82.0 \\
73.0
\end{tabular} & \begin{tabular}{|l|}
24.17 \\
21.37
\end{tabular} & $\begin{array}{l}.570 \\
.430\end{array}$ & $\begin{array}{l}8.890 \\
8.750\end{array}$ & $\begin{array}{l}1559.8 \\
1466.5\end{array}$ & $\begin{array}{l}8.03 \\
8.28\end{array}$ & $\begin{array}{l}156.0 \\
146.7\end{array}$ \\
\hline B20 & $\begin{array}{l}20 \\
20 \\
20\end{array}$ & \begin{tabular}{|l|}
69.0 \\
64.5 \\
59.5
\end{tabular} & $\begin{array}{l}20.26 \\
18.86 \\
17.36\end{array}$ & $\begin{array}{l}.520 \\
.450 \\
.375\end{array}$ & $\begin{array}{l}8.145 \\
8.075 \\
8.000\end{array}$ & $\begin{array}{l}1268.9 \\
1222.1 \\
1172.2\end{array}$ & \begin{tabular}{|l}
7.91 \\
8.05 \\
8.22
\end{tabular} & $\begin{array}{l}126.9 \\
122.2 \\
117.2\end{array}$ \\
\hline
\end{tabular}

$\mathbf{W}=$-Safe load in pounds, uniformly distributed, including weight of heam.

$\mathbf{L}=$ Span, in feet.

$\mathbf{M}=$ Moment of forces, in foot pounds.

I - Allowable Fiber Stress, in Ibs. per square inch.

$\mathbf{S}=$ Section Modulus about axis XX. 


\section{PROPERTIES OF \\ BETHLEHEM I BEAMS.}

\section{COEFFICIENTS OF STRENGTH.}

\begin{tabular}{|c|c|c|c|c|c|c|c|}
\hline & & & & & & & \\
\hline $\begin{array}{l}\text { For Fiber } \\
\text { Stress of } \\
\text { if,000 Llss. } \\
\text { per Sq. In. } \\
\text { UsualBuild } \\
\text { ingCodeRe } \\
\text { quirement. } \\
\text { C }\end{array}$ & $\begin{array}{c}\text { For Fiber } \\
\text { Stress of } \\
12,00(\text { ) Lixs } \\
\text { per Sy. In. } \\
\text { For Moving } \\
\text { Loads. } \\
\text { C' }\end{array}$ & $\begin{array}{c}\text { For Fiber } \\
\text { Stress of } \\
18,(x) \text { I.bs. } \\
\text { per Sq. In. } \\
\text { For Quies. } \\
\text { cent Loads. } \\
\text { C"n }\end{array}$ & $\begin{array}{c}\text { Maxi- } \\
\text { mum } \\
\text { Safe } \\
\text { Shear } \\
\text { on Web, } \\
\text { in } \\
\text { Pounds. }\end{array}$ & $\begin{array}{c}\text { Mo. } \\
\text { ment } \\
\text { of } \\
\text { Inertia. } \\
\text { I' }^{\prime}\end{array}$ & $\begin{array}{l}\text { Radius } \\
\text { of } \\
\text { Ciyra- } \\
\text { tion. } \\
r^{\prime}\end{array}$ & $\begin{array}{l}\text { Section } \\
\text { Modulus. }\end{array}$ & $\begin{array}{l}\text { Section } \\
\text { Num- } \\
\text { ber. }\end{array}$ \\
\hline $3,726,000$ & $2,795,000$ & $4,192,000$ & 103,800 & 165.0 & 2.16 & 31.4 & B30 \\
\hline $3,058,000$ & $2,294,000$ & $3,441,000$ & 89,000 & 131.5 & 2.06 & 26.3 & B28 \\
\hline $2,443,000$ & $1,832,000$ & $2,748,000$ & 75,300 & 101.2 & 1.95 & 21.3 & B26 \\
\hline $2,628,000$ & $1,971,000$ & $2,957,000$ & 104,300 & 132.9 & 2.08 & 27.2 & \\
\hline $2,499,000$ & $1,874,000$ & $2,812,000$ & 95,900 & 124.8 & 2.07 & 25.6 & $\mathrm{~B} 24 \mathrm{~b}$ \\
\hline $2,376,000$ & $1,782,000$ & $2,673,000$ & 89,300 & 117.1 & 2.05 & 24.1 & \\
\hline $2,117,000$ & $1,588,000$ & $2,382,000$ & 75,100 & 91.1 & 1.92 & 19.7 & $\mathrm{~B} 24 \mathrm{a}$ \\
\hline $1,992,000$ & $1,494,00()$ & $2,241,000$ & 93,100 & 78.0 & 1.78 & 17.1 & \\
\hline $1,859,000$ & $1,394,000$ & $2,091,000$ & 54,000 & 74.4 & 1.86 & 16.5 & \\
\hline $1,645,000$ & $1,233,000$ & $1,850,0(x)$ & 62,500 & 65.8 & 1.78 & 15.4 & \\
\hline $1.576,0(0)$ & $1,182,0(x)$ & $1,773,0(x)$ & 58,200 & 62.3 & 1.76 & 14.6 & B:2 \\
\hline $1,503,000$ & $1,127,000$ & $1,690,00()$ & 52,600 & 58.8 & 1.76 & 13.8 & \\
\hline $1,664,000$ & $1,248,00 \times)$ & $1,872,000$ & 102,400 & $79 . ?$ & 1.83 & 18.0 & \\
\hline $1,564,000$ & $1,173,000$ & $1,760,000$ & 64,900 & 75.9 & 1.88 & 17.3 & \\
\hline $1,354,000$ & $1,015,000$ & $1,523,000$ & 88,200 & 51.2 & 1.59 & 12.6 & \\
\hline $1,304,000$ & 977,700 & $1,467,000$ & 69,400 & 49.8 & 1.62 & 12.3 & B20 \\
\hline $1,250,000$ & 937,700 & $1,407,0 \mathrm{CO}$ & 50,000 & 48.3 & 1.66 & 12.1 & \\
\hline
\end{tabular}

$C, C^{\prime}$, and $C^{\prime \prime}-$ Cucflicients given in the table.

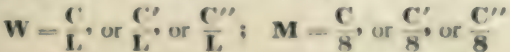

$\mathrm{C}^{\prime}$ or $\mathrm{C}^{\prime}$, or $\mathrm{C}^{\prime \prime}=\mathrm{WL}=8 \mathrm{M}=\frac{2}{\mathrm{P}} \mathrm{S}$ 
BETHLEHEM STEEL COMPANY.

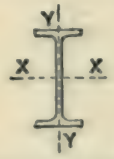

PROPERTIES OF

\section{BETHLEHEM I BEAMS.}

\begin{tabular}{|c|c|c|c|c|c|c|c|c|}
\hline \multirow[b]{2}{*}{$\begin{array}{l}\text { Section } \\
\text { Number. }\end{array}$} & \multirow[b]{2}{*}{$\begin{array}{l}\text { Depth } \\
\text { of } \\
\text { Beam, } \\
\text { Inches. }\end{array}$} & \multirow{2}{*}{$\begin{array}{c} \\
\text { Weight, } \\
\text { Lbs. } \\
\text { per } \\
\text { Foot. }\end{array}$} & \multirow[b]{2}{*}{$\begin{array}{l}\text { Area } \\
\text { of } \\
\text { Section. } \\
\text { Square } \\
\text { Inches. }\end{array}$} & \multirow[b]{2}{*}{$\begin{array}{l}\text { Thick- } \\
\text { ness } \\
\text { of } \\
\text { Web, } \\
\text { Inches. }\end{array}$} & \multirow[b]{2}{*}{$\begin{array}{l}\text { Width } \\
\text { of } \\
\text { Flange, } \\
\text { Inches. }\end{array}$} & \multicolumn{3}{|c|}{ Axis $x x$. } \\
\hline & & & & & & $\begin{array}{c}\text { Moment } \\
\text { of } \\
\text { Inertia. } \\
\text { I }\end{array}$ & $\begin{array}{c}\text { Radius } \\
\text { of } \\
\text { Gyra- } \\
\text { tion. } \\
\\
\text { r }\end{array}$ & $\begin{array}{l}\text { Section } \\
\text { Modulus }\end{array}$ \\
\hline \multirow{4}{*}{$\mathrm{B} 18 \mathrm{a}$} & $181 / 8$ & 74.0 & 21.61 & .440 & 8.770 & 1238.0 & 7.57 & 136.6 \\
\hline & 18 & 69.0 & 20.20 & .420 & 8.750 & 1142.5 & 7.52 & 126.9 \\
\hline & $177 / 8$ & 64.5 & 18.79 & .400 & 8.730 & 1048.5 & 7.47 & 117.3 \\
\hline & 18 & 59.0 & 17.40 & .495 & 7.675 & 883.3 & 7.12 & 98.1 \\
\hline \multirow{3}{*}{ B18 } & 18 & 54.5 & 15.87 & .410 & 7.590 & 842.0 & 7.28 & 93.6 \\
\hline & 18 & 52.0 & 15.24 & .375 & 7.555 & 825.0 & 7.36 & 91.7 \\
\hline & 18 & 49.0 & 14.25 & .320 & 7.500 & 798.3 & 7.48 & 88.7 \\
\hline $\mathrm{B} 15 \mathrm{~b}$ & 15 & 71.5 & 20.95 & .520 & 7.500 & 2 & 6.16 & 106.2 \\
\hline \multirow{2}{*}{$\mathrm{B} 15 \mathrm{a}$} & 15 & 64.0 & 18.81 & .605 & & & & 8.6 \\
\hline & 15 & 54.5 & 15.88 & .410 & 7.000 & 610.0 & 6.20 & 81.3 \\
\hline \multirow{3}{*}{ B15 } & 15 & 46.0 & 13.52 & .440 & 6.810 & 484.8 & 5.99 & 64.6 \\
\hline & 15 & 41.0 & 12.02 & .340 & 6.710 & 456.7 & 6.16 & 60.9 \\
\hline & 15 & 38.5 & 11.27 & .290 & 6.660 & 442.6 & 6.27 & 59.0 \\
\hline $\mathrm{B} 12 \mathrm{a}$ & 12 & 36.5 & 10.61 & .310 & 6.300 & 269.2 & 5.04 & 44.9 \\
\hline \multirow{2}{*}{ B12 } & 12 & 32.0 & 9.44 & .335 & 6.205 & 228.5 & 4.92 & 8.1 \\
\hline & 12 & 28.5 & 8.42 & .250 & 6.120 & .2 & 5.07 & 6.0 \\
\hline \multirow{2}{*}{$\mathrm{B} 10$} & 10 & 28.5 & 8.34 & .390 & 5.990 & 134.6 & 4.02 & 26.9 \\
\hline & 10 & 23.5 & 6.94 & .250 & 5.850 & 122.9 & 4.21 & 24.6 \\
\hline \multirow{2}{*}{ B9 } & 9 & 24.0 & 7.04 & .365 & 5.555 & 92.1 & 3.62 & 20.5 \\
\hline & 9 & 20.5 & 6.01 & .250 & 5.440 & 85.1 & 3.76 & 18.9 \\
\hline \multirow[t]{2}{*}{ B8 } & 8 & 19.5 & 5.78 & .325 & 5.325 & 60.6 & 3.24 & 15.1 \\
\hline & 8 & 17.5 & 5.18 & .250 & 5.250 & 57.4 & 3.33 & 14.3 \\
\hline
\end{tabular}

W - Safe load in pounds, uniformly distributed, including weight of beam.

I. =-Span, in teet.

M-Moment of forces, in foot pounds.

f -Allowable Filer Stress, in lbs. per square inch.

$\mathbf{s}=$ Section Modulus about axis XX. 


\section{PROPERTIES OF \\ BETHLEHEM I BEAMS.}

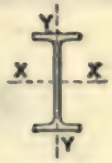

\section{COEFFICIENTS OF STRENGTH}

For Fiber

Stress of For Fiber For Fiber 16,000 Lbs. Stress of Stress of per Sq. In. 12,000 Lhs. 18,000 Lbs. UsualBuild-per Sq. In. per Sq. In. ingCodeRe-For Moving For Quiesquirement.

quirement.

$\frac{\mathbf{C}}{1,458,000} \frac{\mathbf{C}^{\prime}}{1,093,000} \frac{\mathbf{C}^{\prime \prime}}{1,640,000}$ $1,354,0001,016,000 \quad 1,523,000$ $1,251,000 \quad 938,00 \mathrm{C} \quad 1,407,000$ $1,047,000$ 997,900 977,700 946,100

$1,132,000$ 945,600 867,600 689,500 649,400 629,500 478,600 406,200 384,400 287,100 262,200 218,300 201,800 $785,2001,178,000$ $748,400 \quad 1,123,000$ $733,300 \quad 1,100,000$ $709,6001,064,000$ $849,3001,274,000$ $709,2001,064,000$ 650,700 517,100 487,100 472,100 359,000 304,700 457,000 288,300 215,300 323,000 295,000 196,700 163,700 245,600 151,400 161,600 121,200 $153,000 \quad 114,800$ 976,100 775,700 730,600

708,200 538,400 432,500 227,000

\section{Maxi- \\ mum \\ Safe Shear on Web, in Pounds.}

$\overline{66,100}$ 60,800 55,600 78,000 57,500 49,200 36,700 77,900 90,800 54,800 60,000 39,900 30,100 32,200 35,800 22,200 39,000 21,000 32,900 20,100 $181,800 \quad 26,000$ $172,100 \quad 18,900$
AXIS YY.

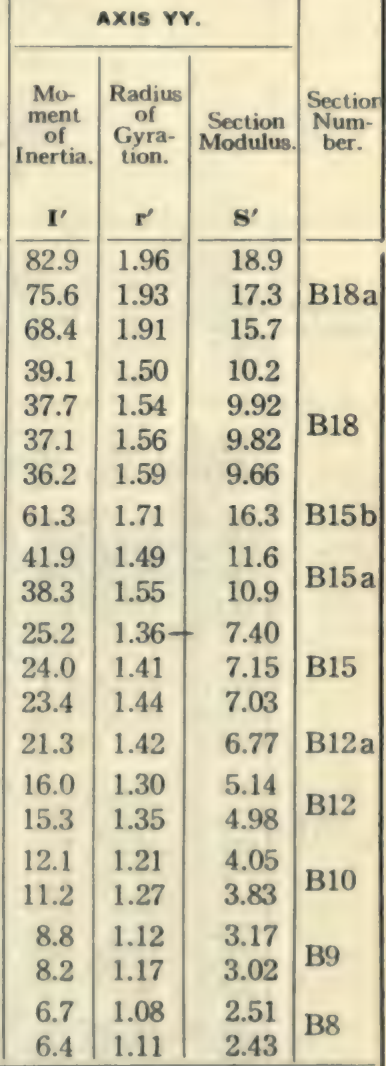

C. C", and $C^{\prime \prime}$ - Coeflicients given in the table.

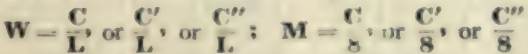

$C$, or $C^{\prime}$, or $C^{\prime \prime}=\mathbf{W L}-8 \mathrm{M}=\frac{2}{3} \mathbf{P} \mathrm{S}$ 
DIMENSIONS AND PROPERTIES OF BETHLEHEM 14" H COLUMNS.

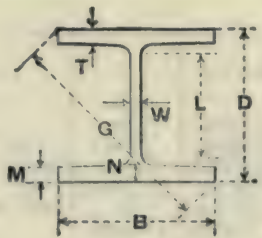

Weight

Section Section.

Number. Lbs.

per

Foot.

84.0

92.0

100.0

107.5

115.5

123.5

131.5

139.0

147.0

155.0

163.0

171.5

179.5

187.5

196.0

204.5

212.0

220.5

228.5

237.0

245.5

254.0

262.5

271.0

279.5

288.5

DIMENSIONS, IN INCHES.

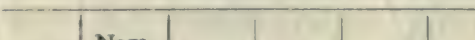

Nom-

inal.

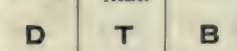

W

M

N G L

$13 \frac{3}{4}$

$\frac{11}{16}$

13.92

13.96

13

14.00

14.04

14.08

14.12

14.16

14.19

14.23

14.27

14.31

14.35

14.39

14.4 .3

14.47

14.51

14.54

14.58

14.62

$157 / 8$

16

\begin{tabular}{l|l}
$161 / 8$ & $17 / 8$
\end{tabular}

$16 \frac{1}{4} 1 \frac{15}{16}$

$163 \% 2$

$16 \frac{8}{2} 2 \frac{1}{16}$

$165 \%$

$163 / 4$

$167 / 8$

14.78

14.82

14.86

14.90

14.66

14.70

14.74

43

.620

.755

$195 / 8$

.817

$193 / 4$

.51

.55

.745

.880

$19 \frac{13}{16}$

.808

.942

.870

1.005

$19 \frac{15}{16}$

.59

.933

1.067

$20 \frac{1}{16}$

.67

.995

1.130

$20 \frac{3}{16}$

.70

1.058

1.120

1.192

$201 / 4$

.74

1.255

.78

1.183

1.317

1.245

1.380

$20^{3} 8$

2015

205

203

.86

$1.308 \quad 1.442$

$\begin{array}{lll}1.370 & 1.505\end{array}$

$1.433 \quad 1.567$

1.495

1.630

$207 / 8$

.90

.94

21

.98

1.558

1.692

1.620

1.755

1.683

1.745

1.817

1.880

2118

$211 / 4$

$213 \%$

217

$21 \%$

$21 \frac{11}{16}$

\begin{tabular}{ll|l|l}
1.808 & 1.942 & $21 \frac{13}{16}$
\end{tabular}

1.8702 .005

1.9332 .067

1.995

2.130

$21 \frac{15}{16}$

221

$22^{3}$

2.058

2.120

2.183

2.192

$22{ }^{5}$ 


\section{DIMENSIONS AND PAOPERTIES OF BETHLEHEM 14" H COLUMNS.}

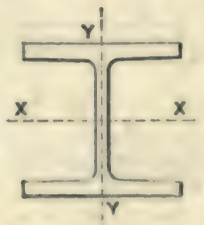

\begin{tabular}{|c|c|c|c|c|c|c|c|c|c|}
\hline \multirow{2}{*}{$\begin{array}{c}\text { Weight } \\
\text { of } \\
\text { Section. } \\
\text { Lbs. } \\
\text { per } \\
\text { Foot. }\end{array}$} & \multirow[b]{2}{*}{$\begin{array}{l}\text { Area } \\
\text { of } \\
\text { Section } \\
\text { Square } \\
\text { Inches }\end{array}$} & \multicolumn{4}{|c|}{$\operatorname{Axis} x$} & \multicolumn{4}{|c|}{ AXIS YY. } \\
\hline & & $\begin{array}{c}\text { Mo-- } \\
\text { ment } \\
\text { of } \\
\text { Inertia. } \\
\mathbf{I} \\
\end{array}$ & $\begin{array}{c}\text { Section } \\
\text { Modu- } \\
\text { lus. } \\
\mathbf{s} \\
\end{array}$ & $\begin{array}{l}\text { Radius } \\
\text { of } \\
\text { Gyra- } \\
\text { tion. } \\
\mathbf{r}\end{array}$ & $\begin{array}{c}\text { Bend- } \\
\text { ing } \\
\text { Factor } \\
\mathbf{k} \\
\end{array}$ & $\begin{array}{c}\text { Mo- } \\
\text { ment } \\
\text { of } \\
\text { Inertia. } \\
\mathbf{I}^{\prime} \\
\end{array}$ & $\begin{array}{l}\text { Section } \\
\text { Modu- } \\
\text { lus. } \\
\mathbf{s}^{\prime} \\
\end{array}$ & $\begin{array}{c}\text { Radius } \\
\text { of } \\
\text { Gyra- } \\
\text { tion. } \\
\mathbf{r}^{\prime} \\
\end{array}$ & $\begin{array}{c}\text { Bend- } \\
\text { ing } \\
\text { Factor } \\
\mathbf{k}^{\prime}\end{array}$ \\
\hline 84.0 & 24.46 & 884.9 & 128.7 & 6.01 & .190 & 294.5 & 42.3 & 3.47 & .578 \\
\hline 92.0 & 26.76 & 976.8 & 140.8 & 6.04 & .190 & 325.4 & 46.6 & 3.49 & .574 \\
\hline 100.0 & 29.06 & 1070.6 & 153.0 & 6.07 & .190 & 356.9 & 51.0 & 3.50 & .570 \\
\hline 7.5 & 31.38 & i1 & 165.2 & 6.10 & .190 & 388.9 & 55.4 & 3.52 & .566 \\
\hline 115.5 & 33.70 & & 177.5 & 6.13 & .190 & 421.3 & 59.9 & 3.54 & .563 \\
\hline .5 & 36.04 & 1364.6 & 189.9 & 6.16 & .190 & 454.4 & 64.4 & $3.5 \overline{5}$ & $.560)$ \\
\hline 131.5 & 38.38 & 1466.7 & 202.3 & 6.1 & .190 & 487.9 & 68.9 & 3.57 & .557 \\
\hline 139.0 & 40.59 & 8.4 & 214.5 & 6.21 & .189 & 520.9 & 73.4 & 3.58 & .553 \\
\hline 147.0 & 42.95 & 4.7 & 227.1 & 6.24 & .189 & 555.4 & 78.1 & 3.60 & .550 \\
\hline .0 & 45.33 & 3.3 & 239.8 & 6.27 & .189 & 500.5 & 83.8 & 3.61 & .548 \\
\hline 16 & 47 & & 252.5 & 6.30 & .189 & 626.1 & 87.5 & 3.62 & .545 \\
\hline 171.5 & 50.11 & 3007.0 & 265.4 & 6.33 & .189 & 662.3 & 92.3 & 3.64 & .543 \\
\hline 5 & 51 & .3 & 2 & 6. & .1 & 699.0 & 97.2 & 5 & .541 \\
\hline .5 & 54.92 & 2339.8 & 291.4 & 6.39 & .18 & $7: 36.3$ & 102.1 & 3.1 & .538 \\
\hline 5.0 & 57.35 & 2359.7 & 304.5 & 6.41 & .18 & 774.2 & 107.0 & 3.68 & .536 \\
\hline & 8 & 1.9 & 3 & 6.4 & .1 & 812.6 & 112.0 & 3.69 & .534 \\
\hline 2 & 07 & 3.3 & 330.6 & 6 & .18 & 849.8 & 116.9 & 3.70 & .531 \\
\hline 5 & 52 & & 1.0 & 6. & .18 & 889.3 & 122.0 & 3 & .529 \\
\hline 228.5 & & & & 6.53 & & 929.4 & 127.1 & 3.72 & .527 \\
\hline 237.0 & 69.45 & 296 & 37 & 6.56 & .187 & 970.0 & 132.3 & 3.74 & .525 \\
\hline & 71.94 & & 384.7 & 6.59 & .187 & 1011.3 & 137.6 & 3.75 & .523 \\
\hline 254.0 & 74.43 & 3262.7 & 398.5 & 6.62 & .187 & 1053.2 & 142.9 & 3.76 & .521 \\
\hline $36 ? .5$ & 76.93 & 3402.1 & 412 & 6. & .18 & 1095 & 148 & 3.77 & .519 \\
\hline 10 & 79.44 & 3544.1 & 426.4 & 6. & 18 & 1138.7 & 153.7 & & .517 \\
\hline & & & & 6. & .18 & 1182.4 & 159.1 & 3.80 & .515 \\
\hline 288.5 & 84.50 & 36.1 & 4.7 & 6.74 & .186 & $\mid 1226.7$ & 164.7 & 3.81 & .513 \\
\hline
\end{tabular}


DIMENSIONS AND PROPERTIES OF BETHLEHEM 12" H COLUMNS.

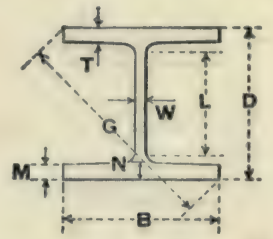

Section

Weight Number.

\begin{tabular}{|c|c|c|}
\hline Number. & $\begin{array}{l}\text { Lbs. } \\
\text { per } \\
\text { Foot. }\end{array}$ & D \\
\hline & 65.5 & $113 / 4$ \\
\hline & 72.5 & $117 / 8$ \\
\hline 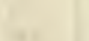 & 79.0 & 12 \\
\hline & 85.5 & $121 / 8$ \\
\hline & 92.5 & $121 / 4$ \\
\hline & 99.5 & $123 / 8$ \\
\hline & 106.0 & $121 / 2$ \\
\hline & 113.0 & $125 / 8$ \\
\hline H12 & 119.5 & $123 / 4$ \\
\hline 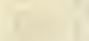 & 126.5 & $127 / 8$ \\
\hline & 133.5 & 13 \\
\hline 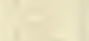 & 140.5 & $131 / 8$ \\
\hline & 147.5 & $13 \frac{1}{4}$ \\
\hline 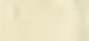 & 154.5 & $13 \frac{3}{8}$ \\
\hline & 162.0 & $131 / 2$ \\
\hline & 169.0 & $135 / 8$ \\
\hline 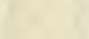 & 176.0 & $133 / 4$ \\
\hline & 183.0 & $137 / 8$ \\
\hline & 190.0 & 14 \\
\hline
\end{tabular}

DIMENSIONS, IN INCHES.

Nominal.

$\begin{array}{lll}T & B & W\end{array}$

\begin{tabular}{l|l}
$5 / 8$ & 11.92
\end{tabular}

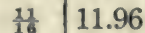

$3 / 4 \quad 12.00$

1312.04

$7 / 8$

12.08

12.08

12.12

12.16

1

$1 \frac{1}{16}$

12.20

$11 / 8$

12.23

$1 \frac{3}{16}$

$11 / 4$

12.27

12.31

$1 \frac{5}{16} \quad 12.35$

$13 / 8 \quad 12.39$

$1 \frac{7}{16} \quad 1243$

$11 / 2$

12.47

1 으 12.51

$15 / 8$

1255

12.551 .02

12.58

$1 \frac{1}{16}$

$13 / 4$

12.62 


\section{DIMENSIONS AND PROPEATIES OF} BETHLEHEM 12" H COLUMNS.

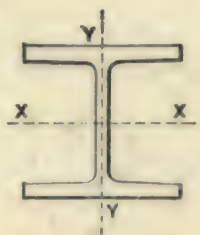

\begin{tabular}{|c|c|c|c|c|c|c|c|c|c|}
\hline \multirow{2}{*}{$\begin{array}{l}\text { Weight } \\
\text { of } \\
\text { Section, } \\
\text { Lbs. } \\
\text { per } \\
\text { Foot. }\end{array}$} & \multirow{2}{*}{$\begin{array}{c}\text { Area } \\
\text { of } \\
\text { Section, } \\
\text { Square } \\
\text { Inches. }\end{array}$} & \multicolumn{4}{|c|}{ AXIS. XX. } & \multicolumn{4}{|c|}{ AXIS YY. } \\
\hline & & $\begin{array}{c}\text { Mo- } \\
\text { ment } \\
\text { of } \\
\text { Inertia. } \\
I\end{array}$ & \begin{tabular}{|c|} 
Section \\
Modu- \\
lus. \\
$\mathbf{S}$ \\
\end{tabular} & $\begin{array}{c}\text { Radius } \\
\text { of } \\
\text { Gyra- } \\
\text { tion. } \\
\mathbf{r} \\
\end{array}$ & $\begin{array}{c}\begin{array}{c}\text { Bend- } \\
\text { ing } \\
\text { Factor } \\
\mathbf{k}\end{array} \\
\end{array}$ & $\begin{array}{c}\text { Mo- } \\
\text { ment } \\
\text { of } \\
\text { Inertia. } \\
\mathbf{I}^{\prime}\end{array}$ & $\begin{array}{c}\text { Section } \\
\text { Modu- } \\
\text { lus. } \\
\mathbf{S}^{\prime} \\
\end{array}$ & $\begin{array}{c}\text { Radius } \\
\text { of } \\
\text { Gyra- } \\
\text { tion. } \\
\mathbf{r}^{\prime}\end{array}$ & $\begin{array}{c}\text { Bend- } \\
\text { ing } \\
\text { Factor. } \\
\mathbf{k}^{\prime}\end{array}$ \\
\hline 65.5 & 19.00 & 499.0 & 84.9 & 5.13 & .224 & 168.6 & 28.3 & 2.98 & .672 \\
\hline 72.5 & 20.96 & 556.6 & 93.7 & 5.15 & .224 & 188.2 & 31.5 & 3.00 & .666 \\
\hline 79.0 & 22.94 & 61 & 102.6 & 5.18 & .224 & 208.1 & 34.7 & 3.01 & 661 \\
\hline 85.5 & 24.92 & 676.1 & 111.5 & 5.21 & .224 & 228.5 & 37.9 & 3.03 & .657 \\
\hline 92.5 & 26.92 & 738.1 & 120.5 & 5.24 & .223 & 249.2 & 41.3 & 3.04 & .652 \\
\hline 99.5 & 28.92 & 801.7 & 129.6 & 5.27 & .223 & 270.1 & 44.6 & 3.06 & .649 \\
\hline 106.0 & 30.94 & 8 & 138.6 & 5.30 & .223 & 7 & 4 & 3.07 & .645 \\
\hline 113.0 & 32.96 & 933.4 & 147.9 & 5.33 & .223 & 313.6 & 51.4 & 3.08 & .641 \\
\hline 119.5 & 34.87 & 1000.0 & 156.9 & 5.36 & .222 & 335.0 & 54.8 & 3.10 & .637 \\
\hline 126.5 & 36.91 & 1069.8 & 166.2 & 5.38 & .222 & 357.7 & 58.3 & 3.11 & .633 \\
\hline 133.5 & 38.97 & 1141.3 & 175.6 & 5.41 & .222 & 380.7 & 61.9 & 3.13 & .630 \\
\hline 140.5 & 41.03 & 1214.5 & 185.0 & 5.44 & .222 & 404.1 & 65.4 & 3.14 & .627 \\
\hline 147.5 & 43.10 & 1289.4 & 194.6 & 5.47 & .221 & 428.0 & 69.1 & 3.15 & .624 \\
\hline 154.5 & 45.19 & 1366.0 & 204.3 & 5.50 & .221 & 452.2 & 72.8 & 3.16 & .621 \\
\hline 162.0 & 47.28 & 1444.3 & 214.0 & 5.53 & .221 & 477.0 & 76.5 & 3.18 & .618 \\
\hline 169.0 & 49.38 & 1524.4 & 223.8 & 5.56 & .221 & 502.1 & 80.3 & 3.19 & .615 \\
\hline 176.0 & 51.50 & 1606.3 & 233.6 & 5.59 & .220 & 527.6 & 84.1 & 3.20 & .612 \\
\hline 183.0 & 53.48 & 16 & 243.3 & 5.62 & .220 & 552.2 & 87.8 & 3.21 & .609 \\
\hline 190.0 & 55.62 & 1773.4 & 253.3 & 5.65 & .220 & 578.6 & 91.7 & 3.23 & .607 \\
\hline
\end{tabular}


BETHLEHEM STEEL COMPANY.

DIMENSIONS AND PROPERTIES OF

BETHLEHEM 10" H COLUMNS.

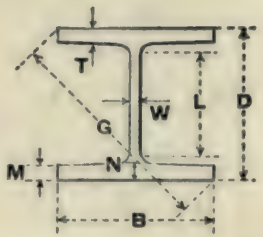

\begin{tabular}{|c|c|c|c|c|c|c|c|c|c|}
\hline \multirow[b]{2}{*}{$\begin{array}{l}\text { Section } \\
\text { Number. }\end{array}$} & \multirow{2}{*}{$\begin{array}{c}\text { Weight } \\
\text { of } \\
\text { Section, } \\
\text { Lbs. } \\
\text { per } \\
\text { Foot. }\end{array}$} & \multicolumn{8}{|c|}{ DIMENSIONS, IN INCHES. } \\
\hline & & D & $\begin{array}{c}\text { Nom- } \\
\text { inal. } \\
T\end{array}$ & B & $\mathbf{w}$ & M & $\mathbf{N}$ & G & L \\
\hline & 49.5 & $97 / 8$ & $\frac{9}{16}$ & 9.97 & .36 & .514 & .611 & $14 \frac{1}{16}$ & \\
\hline & 55.0 & 10 & $5 / 8$ & 10.00 & .39 & .577 & .673 & $14 \frac{3}{16}$ & \\
\hline & 60.5 & $101 / 8$ & $\frac{11}{16}$ & 10.04 & .43 & .639 & .736 & $14 \frac{5}{16}$ & \\
\hline & 66.0 & $101 / 4$ & $3 / 4$ & 10.08 & .47 & .702 & .798 & $143 / 8$ & \\
\hline & 72.0 & $10^{3} / 8$ & $\frac{13}{16}$ & 10.12 & .51 & .764 & .861 & $14 \frac{1}{2}$ & \\
\hline & 77.5 & $10 \frac{1}{2}$ & $7 / 8$ & 10.16 & .55 & .827 & .923 & 1458 & N \\
\hline & 83.5 & $105 \%$ & $\frac{15}{16}$ & 10.20 & .59 & .889 & .986 & $14^{3} / 4$ & 11 \\
\hline$H$ & 89.0 & $10^{3} ;$ & 1 & 10.24 & .63 & .952 & 1.048 & $147 / 8$ & 范 \\
\hline 1110 & 95.0 & $107 / 8$ & $1 \frac{1}{16}$ & 10.28 & .67 & 1.014 & 1.111 & 15 & \\
\hline & 100.5 & 11 & $11 / 8$ & 10.31 & .70 & 1.077 & 1.173 & $151 / 8$ & 8 \\
\hline & 106.5 & $111 / 8$ & $1 \frac{3}{16}$ & 10.35 & .74 & 1.139 & 1.236 & $15 \frac{3}{16}$ & \\
\hline & 112.0 & $111 / 4$ & $11 / 4$ & 10.39 & .78 & 1.202 & 1.298 & $15 \frac{5}{16}$ & \\
\hline & 118.0 & $113 / 8$ & $1 \frac{5}{16}$ & 10.43 & .82 & 1.264 & 1.361 & $15 \frac{7}{16}$ & \\
\hline & 124.0 & $111 / 2$ & $13 / 8$ & 10.47 & .86 & 1.327 & 1.423 & $15_{16}^{9}$ & \\
\hline & 130.0 & $115 / 8$ & $1 \frac{7}{16}$ & 10.51 & .90 & 1.389 & 1.486 & $151 \frac{1}{16}$ & \\
\hline & 136.5 & $113 / 4$ & $11 / 2$ & 10.55 & .94 & 1.452 & 1.548 & $151 \frac{3}{16}$ & \\
\hline
\end{tabular}


DIMENSIONS AND PROPERTIES OF

BETHLEHEM 10" H COLUMNS.

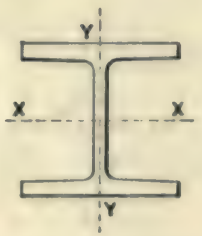

\begin{tabular}{|c|c|c|c|c|c|c|c|c|c|}
\hline \multirow[b]{2}{*}{$\begin{array}{c}\text { Weight } \\
\text { of } \\
\text { Section, } \\
\text { Lbs. } \\
\text { per } \\
\text { Foot. }\end{array}$} & \multirow[b]{2}{*}{$\begin{array}{c}\text { Area } \\
\text { of } \\
\text { Section, } \\
\text { Square } \\
\text { Inches. }\end{array}$} & \multicolumn{4}{|c|}{ AXIS XX. } & \multicolumn{4}{|c|}{ AXIS YY. } \\
\hline & & $\begin{array}{c}\text { Mo- } \\
\text { ment } \\
\text { of } \\
\text { Inertia. } \\
\text { I }\end{array}$ & $\begin{array}{c}\text { Section } \\
\text { Modu- } \\
\text { lus. } \\
\mathbf{s}\end{array}$ & $\begin{array}{c}\text { Radius } \\
\text { of } \\
\text { Gyra- } \\
\text { tion. } \\
r\end{array}$ & $\begin{array}{c}\text { Bend- } \\
\text { ing } \\
\text { Factor. } \\
\mathbf{k}\end{array}$ & $\begin{array}{c}\text { Mo- } \\
\text { ment } \\
\text { of } \\
\text { Inertia. } \\
I^{\prime}\end{array}$ & $\begin{array}{c}\text { Section } \\
\text { Modu- } \\
\text { lus. } \\
\mathbf{s}^{\prime}\end{array}$ & $\begin{array}{c}\text { Radius } \\
\text { of } \\
\text { Gyra- } \\
\text { tion. } \\
\mathbf{r}^{\prime}\end{array}$ & $\begin{array}{c}\begin{array}{c}\text { Bend- } \\
\text { ing } \\
\text { Factor. }\end{array} \\
\mathbf{k}^{\prime}\end{array}$ \\
\hline 49.5 & 14.37 & 263.5 & 53.4 & 4.28 & .269 & 89.1 & 17.9 & 2.49 & .804 \\
\hline 55.0 & 15.91 & 296.8 & 59.4 & 4.32 & .268 & 100.4 & 20.1 & 2.51 & .792 \\
\hline 60.5 & 17.57 & 331.9 & 65.6 & 4.35 & .268 & 112.2 & 22.3 & 2.53 & .786 \\
\hline 66.0 & 19.23 & 368.0 & 71.8 & 4.37 & .268 & 124.2 & 24.6 & 2.54 & .780 \\
\hline 72.0 & 20.91 & 405.2 & 78.1 & 4.40 & .268 & 136.5 & 27.0 & 2.56 & .775 \\
\hline 77.5 & 22.59 & 443.6 & 84.5 & 4.43 & .267 & 149.1 & 29.4 & 2.57 & .770 \\
\hline 83.5 & 24.29 & 483.0 & 90.9 & 4.46 & .267 & 162.0 & 31.8 & 2.58 & .765 \\
\hline 89.0 & 25.99 & 523.5 & 97.4 & 4.49 & .267 & 175.1 & 34.2 & 2.60 & .760 \\
\hline 95.0 & 27.71 & 565.2 & 103.9 & 4.52 & .267 & 188.6 & 36.7 & 2.61 & .755 \\
\hline 100.5 & 29.32 & 607.0 & 110.4 & 4.55 & .266 & 201.7 & 39.1 & 2.62 & .749 \\
\hline 106.5 & 31.06 & 651.0 & 117.0 & 4.58 & .265 & 215.6 & 41.7 & 2.64 & .746 \\
\hline 112.0 & 32.80 & 696.2 & 123.8 & 4.61 & .265 & 229.9 & 44.3 & 2.65 & .741 \\
\hline 118.0 & 34.55 & 742.7 & 130.6 & 4.64 & .265 & 244.4 & 46.9 & 2.66 & .737 \\
\hline 124.0 & 36.32 & 790.4 & 137.5 & 4.67 & .264 & 259.3 & 49.5 & 2.67 & .733 \\
\hline 130.0 & 38.09 & 839.4 & 144.4 & 4.69 & .264 & 274.5 & 52.2 & 2.68 & .729 \\
\hline 136.5 & 39.88 & 889.7 & 151.4 & 4.72 & .263 & 289.9 & 5.5 .0 & 2.70 & .726 \\
\hline
\end{tabular}


BETHLEHEM STEEL COMPANY.

\section{DIMENSIONS AND PROPERTIES OF}

BETHLEHEM 8" H COLUMNS.

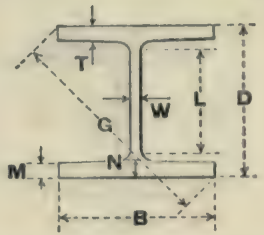

Section Section,

Number. Lbs.

per

Foot.

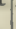

H8

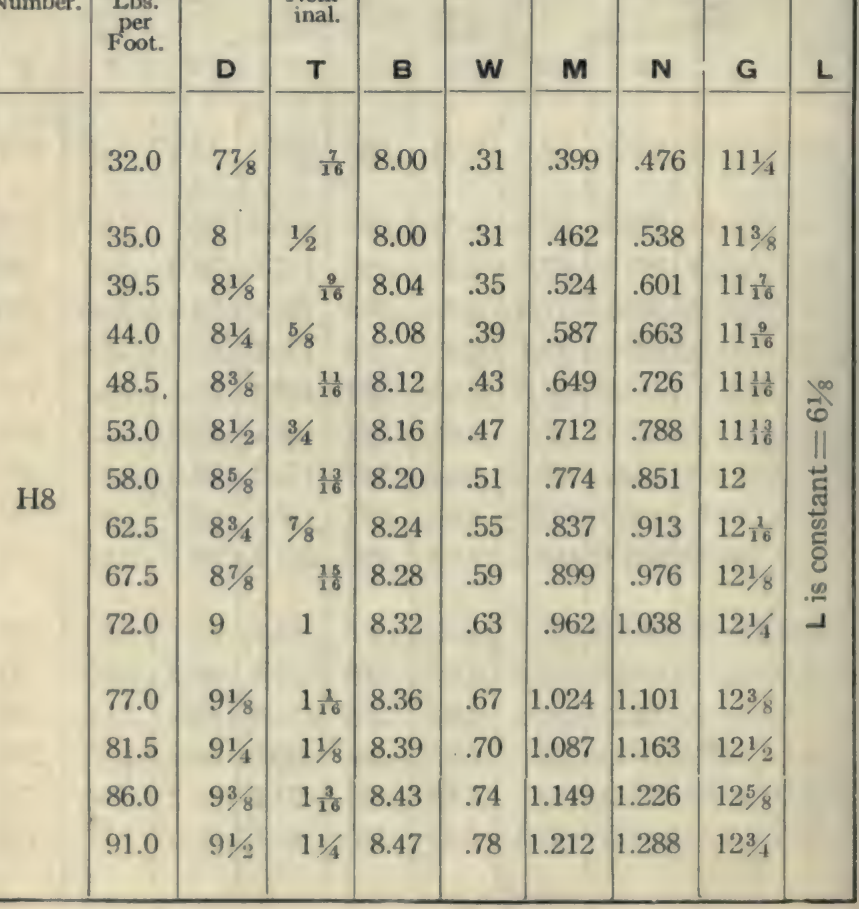


DIMENSIONS AND PROPERTIES OF

\section{BETHLEHEM 8" H COLUMNS.}

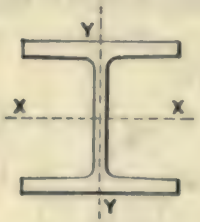

\begin{tabular}{|c|c|c|c|c|c|c|c|c|c|}
\hline \multirow{2}{*}{$\begin{array}{c}\text { Weight } \\
\text { of } \\
\text { Section. } \\
\text { Lbs. } \\
\text { per } \\
\text { Foot. }\end{array}$} & \multirow{2}{*}{$\begin{array}{l}\text { Area } \\
\text { of } \\
\text { Section, } \\
\text { Square } \\
\text { Inches. }\end{array}$} & \multicolumn{4}{|c|}{ AXIS XX. } & \multicolumn{4}{|c|}{ AXIS YY. } \\
\hline & & $\begin{array}{c}\text { Mo- } \\
\text { ment } \\
\text { of } \\
\text { Inertia. } \\
\text { I }\end{array}$ & $\begin{array}{c}\text { Section } \\
\text { Modu- } \\
\text { lus. } \\
\mathbf{s}\end{array}$ & $\begin{array}{c}\text { Radius } \\
\text { of } \\
\text { Gyra- } \\
\text { tion. } \\
\mathbf{r}\end{array}$ & $\begin{array}{c}\text { Bend- } \\
\text { ing } \\
\text { Factor. } \\
\mathbf{k}\end{array}$ & $\begin{array}{c}\text { Mo- } \\
\text { ment } \\
\text { of } \\
\text { Inertia. } \\
I^{\prime}\end{array}$ & $\begin{array}{c}\text { Section } \\
\text { Modu- } \\
\text { lus. } \\
\text { S }^{\prime}\end{array}$ & $\begin{array}{c}\text { Radius } \\
\text { of } \\
\text { Gyra- } \\
\text { tion. } \\
\mathbf{r}^{\prime}\end{array}$ & $\begin{array}{c}\text { Bend- } \\
\text { ing } \\
\text { Factor. } \\
\mathbf{k}^{\prime}\end{array}$ \\
\hline 32.0 & 9.17 & 105.7 & 26.9 & 3.40 & .342 & 35.8 & 8.9 & 1.98 & 1.025 \\
\hline 35.0 & 10.17 & 121.5 & 30.4 & 3.46 & .335 & 41.1 & 10.3 & 2.01 & .990 \\
\hline 39.5 & 11.50 & 139.5 & 34.3 & 3.48 & .335 & 47.2 & 11.7 & 2.03 & .979 \\
\hline 44.0 & 12.83 & 158.3 & 38.4 & 3.51 & .334 & 53.4 & 13.2 & 2.04 & .971 \\
\hline 48.5 & 14.18 & 177.7 & 42.4 & 3.54 & .334 & 59.8 & 14.7 & 2.05 & .963 \\
\hline 53.0 & 15.53 & 197.8 & 46.5 & 3.57 & .334 & 66.3 & 16.3 & 2.07 & .956 \\
\hline 58.0 & 16.90 & 218.6 & 50.7 & 3.60 & .333 & 73.1 & 17.8 & 2.08 & .948 \\
\hline 62.5 & 18.27 & 240.2 & 54.9 & 3.63 & .333 & 80.0 & 19.4 & 2.09 & .941 \\
\hline 67.5 & 19.66 & 262.5 & 59.2 & 3.65 & .332 & 87.1 & 21.0 & 2.11 & .934 \\
\hline 72.0 & 21.05 & 285.6 & 63.5 & 3.68 & .332 & 94.4 & 22.7 & 2.12 & .928 \\
\hline 77.0 & 22.46 & 309.5 & 67.8 & 3.71 & .331 & 101.9 & 24.4 & 2.13 & .921 \\
\hline 81.5 & 23.78 & 333.5 & 72.1 & 3.75 & .330 & 109.2 & 26.0 & 2.14 & .914 \\
\hline 86.0 & 25.20 & 359.0 & 76.6 & 3.77 & .329 & 117.1 & 27.8 & 2.16 & .907 \\
\hline 91.0 & 26.64 & 385.3 & 81.1 & 3.80 & .328 & 125.1 & 29.6 & 2.17 & .902 \\
\hline
\end{tabular}


B ETHLEHEM STEEL COMPANY.

\section{DIMENSIONS AND PROPERTIES OF \\ BETHLEHEM COLUMNS. \\ 14" AND 12" \\ SUPPLEMENTARY SECTIONS.}

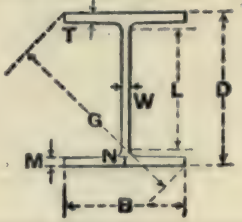

\begin{tabular}{|c|c|c|c|c|c|c|c|c|c|}
\hline \multirow{2}{*}{$\begin{array}{l}\text { Section } \\
\text { Num- } \\
\text { ber. }\end{array}$} & \multirow{2}{*}{$\begin{array}{l}\text { Weight } \\
\text { of } \\
\text { Section, } \\
\text { Lbs. } \\
\text { per } \\
\text { Foot. }\end{array}$} & \multicolumn{8}{|c|}{ DIMENSIONS, IN INCHES. } \\
\hline & & $\begin{array}{c}\text { Nom- } \\
\text { inal } \\
\text { D }\end{array}$ & $\begin{array}{c}\text { Nom- } \\
\text { inal } \\
T\end{array}$ & B & W & M & $\mathbf{N}$ & G & L \\
\hline \multirow{4}{*}{$H \frac{14}{8}$} & 43.0 & $13 \frac{3}{8}$ & $1 / 2$ & 8.00 & .31 & .491 & .567 & $15 \frac{9}{16}$ & $11 \frac{1}{16}$ \\
\hline & 48.0 & $131 / 2$ & $\frac{9}{16}$ & 8.04 & .35 & .553 & .630 & $15 \frac{11}{16}$ & $11 \frac{1}{16}$ \\
\hline & 53.5 & $135 / 8$ & $5 / 8$ & 8.08 & .39 & .616 & .692 & $151 \%$ & 1116 \\
\hline & 58.5 & $13 \frac{3}{4}$ & $\frac{11}{16}$ & 8.12 & .43 & .678 & .755 & 16 & $11 \frac{1}{16}$ \\
\hline \multirow{4}{*}{$H \frac{14}{10}$} & 55.0 & $131 / 2$ & $\frac{9}{16}$ & 10.00 & .35 & .533 & .630 & $16 \frac{13}{16}$ & $11 \frac{1}{16}$ \\
\hline & 61.5 & $135 / 8$ & $5 / 8$ & 10.04 & .39 & .596 & .692 & $16 \frac{15}{16}$ & $11 \frac{1}{16}$ \\
\hline & 67.5 & $133 / 4$ & $\frac{11}{16}$ & 10.08 & .43 & .658 & .755 & $17 \frac{1}{16}$ & $11 \frac{x}{16}$ \\
\hline & 73.5 & $137 / 8$ & $3 / 4$ & 10.12 & .47 & .721 & .817 & $17 \frac{3}{16}$ & $11 \frac{1}{16}$ \\
\hline \multirow{4}{*}{$\mathrm{H} \frac{14}{12}$} & 69.0 & 1: & $5 / 8$ & 0 & .39 & .576 & .692 & $181 / 8$ & $11 \frac{2}{16}$ \\
\hline & 76.0 & $133 / 4$ & $\frac{11}{16}$ & 12.04 & .43 & .639 & .755 & $181 / 4$ & $11 \frac{1}{16}$ \\
\hline & 83.0 & $137 / 8$ & $3 / 4$ & 12.08 & .47 & .701 & .817 & $183 / 8$ & $11 \frac{1}{16}$ \\
\hline & 90.0 & 14 & $\frac{13}{16}$ & 12.12 & .51 & .764 & .880 & $181 / 2$ & $11 \frac{1}{16}$ \\
\hline \multirow{4}{*}{$H \frac{12}{8}$} & 40.5 & $111 / 2$ & $1 / 2$ & 8.00 & .31 & .481 & .558 & 14 & $9 \frac{3}{16}$ \\
\hline & 45.5 & $115 / 8$ & $\frac{9}{16}$ & 8.04 & .35 & .543 & .620 & $141 / 8$ & $9 \frac{3}{16}$ \\
\hline & 50.5 & $113 / 4$ & $5 / 8$ & 8.08 & .39 & .606 & .683 & $141 / 4$ & $9 \frac{3}{16}$ \\
\hline & 55.0 & $117 / 8$ & $\frac{11}{16}$ & 8.12 & .43 & .668 & .745 & $143 / 8$ & $9 \frac{3}{16}$ \\
\hline \multirow{4}{*}{$H \frac{12}{10}$} & 52.5 & $115 / 8$ & $\frac{9}{16}$ & 10.00 & .35 & .524 & .620 & $15 \frac{3}{16}$ & $9 \frac{3}{16}$ \\
\hline & 58.0 & $113 / 4$ & $5 / 8$ & 10.04 & .39 & .586 & .683 & $15 \frac{7}{16}$ & $9 \frac{3}{16}$ \\
\hline & 64.0 & $117 / 8$ & $\frac{11}{16}$ & 10.08 & .43 & .649 & .745 & $15 \frac{9}{16}$ & $9 \frac{3}{16}$ \\
\hline & 70.0 & 12 & $3 / 4$ & 10.12 & .47 & .711 & .808 & $15 \frac{11}{16}$ & $9 \frac{3}{16}$ \\
\hline
\end{tabular}




\section{DIMENSIONS AND PROPERTIES OF BETHLEHEM COLUMNS. $14^{\prime \prime}$ AND $12^{\prime \prime}$ SUPPLEMENTARY SECTIONS.

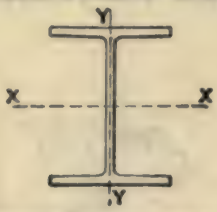

\begin{tabular}{|c|c|c|c|c|c|c|c|c|c|}
\hline \multirow[b]{2}{*}{$\begin{array}{l}\text { Weight } \\
\text { of } \\
\text { Section, } \\
\text { Lbs. } \\
\text { per } \\
\text { Foot. }\end{array}$} & \multirow[b]{2}{*}{$\begin{array}{c}\text { Area } \\
\text { of } \\
\text { Section, } \\
\text { Square } \\
\text { Inches. }\end{array}$} & \multicolumn{4}{|c|}{ AxIs XX. } & \multicolumn{4}{|c|}{ Axis YY. } \\
\hline & & $\begin{array}{c}\text { Mo- } \\
\text { ment } \\
\text { of } \\
\text { Inertia. } \\
\mathbf{I} \\
\end{array}$ & $\begin{array}{c}\text { Section } \\
\text { Modu- } \\
\text { lus. } \\
\mathbf{S} \\
\end{array}$ & $\begin{array}{c}\text { Radius } \\
\text { of } \\
\text { Gyra- } \\
\text { tion, } \\
\text { Inches. } \\
\mathbf{r} \\
\end{array}$ & $\begin{array}{c}\begin{array}{c}\text { Bend- } \\
\text { ing } \\
\text { Factor }\end{array} \\
\mathbf{k} \\
\end{array}$ & $\begin{array}{c}\text { Mo- } \\
\text { ment } \\
\text { of } \\
\text { Inertia. } \\
I^{\prime} \\
\end{array}$ & $\begin{array}{c}\text { Section } \\
\text { Modu- } \\
\text { lus. } \\
\mathbf{S}^{\prime} \\
\end{array}$ & $\begin{array}{c}\text { Radius } \\
\text { of } \\
\text { Gyra- } \\
\text { tion, } \\
\text { Inches. } \\
\mathbf{r}^{\prime}\end{array}$ & $\begin{array}{l}\text { Bend- } \\
\text { ing } \\
\text { Factor } \\
\mathbf{k}^{\prime}\end{array}$ \\
\hline 43.0 & 12.28 & 397.6 & 59.5 & 5.69 & .207 & 43.6 & 10.9 & 1.88 & 1.127 \\
\hline 48.0 & 13.82 & 450.9 & 66.8 & 5.71 & .207 & 49.7 & 12.4 & 1.90 & 1.118 \\
\hline 53.5 & 15.37 & 505.6 & 74.2 & 5.74 & .207 & 56.0 & 13.9 & 1.91 & 1.110 \\
\hline 58.5 & 16.93 & 561.6 & 81.7 & 5.76 & .207 & 62.4 & 15.4 & 1.92 & 1.102 \\
\hline 55.0 & 15.95 & 540.4 & 80.1 & 5.82 & .199 & 93.1 & 18.6 & 2.42 & .857 \\
\hline 61.5 & 17.75 & 606.3 & 89.0 & 5.84 & .199 & 104.8 & 20.9 & 2.43 & .850 \\
\hline 67.5 & 19.55 & 673.7 & 98.0 & 5.87 & .200 & 116.8 & 23.2 & 2.44 & .844 \\
\hline 73.5 & 21.37 & 742.7 & 107.1 & 5.90 & .200 & 129.0 & 25.5 & 2.46 & .838 \\
\hline 69.0 & 20.04 & 704.0 & 103.3 & 5.93 & .194 & 174.7 & 29.1 & 2.95 & .689 \\
\hline 76.0 & 22.09 & 782.9 & 113.9 & 5.95 & .194 & 194.7 & 32.3 & 2.97 & .683 \\
\hline 83.0 & 24.15 & 863.6 & 124.5 & 5.98 & .194 & 215.0 & 35.6 & 2.98 & .678 \\
\hline 90.0 & 26.22 & 946.1 & 135.2 & 6.01 & .194 & 235.8 & 38.9 & 3.00 & .674 \\
\hline 40.5 & 11.55 & 280.1 & 48.7 & 4.92 & .2 & 0 & 7 & 1.92 & 1.081 \\
\hline 45.5 & 13.02 & 318.8 & 54.8 & 4.95 & .237 & 48.8 & 12.1 & 1.94 & 1.071 \\
\hline 50.5 & 14.49 & 358.5 & 61.0 & 4.97 & .237 & 55.1 & 13.6 & 1.95 & 1.063 \\
\hline 55.0 & 15.98 & 399.3 & 67.3 & 5.00 & .238 & 61.5 & 15.2 & 1.96 & 1.055 \\
\hline 52.5 & 15.11 & 383.2 & 65.9 & 5.04 & $.2 \angle 9$ & 91.5 & 18.3 & 2.46 & .820 \\
\hline 58.0 & 16.83 & 431.3 & 73.4 & 5.06 & .229 & 103.2 & 20.5 & 2.48 & .819 \\
\hline 64.0 & 18.55 & 480.6 & 80.9 & 5.09 & 229 & 115.1 & 22.8 & 2.49 & .813 \\
\hline 70.0 & 20.30 & 531.3 & 88.5 & 5.12 & .229 & 127.3 & 25.2 & 2.50 & .807 \\
\hline
\end{tabular}


BETHLEHEM STEEL COMPANY.

\section{DIMENSIONS AND PROPERTIES OF \\ BETHLEHEM COLUMNS. \\ 10", 8" AND 6" \\ SUPPLEMENTARY SECTIONS.}

\begin{tabular}{|c|c|c|c|c|c|c|c|c|c|}
\hline \multirow{2}{*}{$\begin{array}{l}\text { Section } \\
\text { Num- } \\
\text { ber. }\end{array}$} & \multirow{2}{*}{$\begin{array}{l}\text { Weight } \\
\text { of } \\
\text { Section, } \\
\text { Lbs. } \\
\text { per } \\
\text { Foot. }\end{array}$} & \multicolumn{8}{|c|}{ DIMENSIONS, IN INCHES. } \\
\hline & & $\begin{array}{c}\text { Nom- } \\
\text { inal } \\
\text { D }\end{array}$ & $\begin{array}{c}\text { Nom- } \\
\text { inal } \\
\mathbf{T}\end{array}$ & $\mathbf{B}$ & W & M & $\mathbf{N}$ & G & L \\
\hline$H \frac{10}{8}$ & $\begin{array}{l}33.5 \\
38.0 \\
42.5 \\
47.5\end{array}$ & $\begin{array}{c}95 / 8 \\
93 / 4 \\
97 / 8 \\
10\end{array}$ & $\begin{array}{l}\frac{7}{16} \\
1 / 2 \\
\frac{9}{16} \\
5 / 8\end{array}$ & $\begin{array}{l}8.00 \\
8.03 \\
8.07 \\
8.11\end{array}$ & $\begin{array}{l}.28 \\
.31 \\
.35 \\
.39\end{array}$ & $\begin{array}{r}.408 \\
.471 \\
.533 \\
.596\end{array}$ & $\begin{array}{l}.486 \\
.548 \\
.611 \\
.673\end{array}$ & $\begin{array}{l}121 / 2 \\
125 / 8 \\
123 / 4 \\
127 / 8\end{array}$ & $\begin{array}{l}7 \frac{11}{16} \\
7 \frac{11}{16} \\
7 \frac{11}{16} \\
7 \frac{1}{16}\end{array}$ \\
\hline$H \frac{8}{6.5}$ & $\begin{array}{l}23.5 \\
27.0 \\
30.5 \\
34.5\end{array}$ & $\begin{array}{l}73 / 4 \\
77 / 8 \\
8 \\
81 / 8\end{array}$ & $\begin{array}{l}3 / 8 \\
\frac{7}{16} \\
1 / 2 \\
\frac{9}{16}\end{array}$ & $\begin{array}{l}6.50 \\
6.53 \\
6.56 \\
6.60\end{array}$ & $\begin{array}{l}.25 \\
.28 \\
.31 \\
.35\end{array}$ & $\begin{array}{l}.351 \\
.413 \\
.476 \\
.538\end{array}$ & $\begin{array}{l}.413 \\
.476 \\
.538 \\
.601\end{array}$ & $\begin{array}{l}10^{1 / 8} \\
101 / 4 \\
10^{3} / 8 \\
10^{1 / 2}\end{array}$ & $\begin{array}{l}61 / 8 \\
61 / 8 \\
61 / 8 \\
61 / 8\end{array}$ \\
\hline H6 & $\begin{array}{l}20.0 \\
23.0 \\
26.5 \\
30.0 \\
33.5 \\
37.0 \\
40.5\end{array}$ & $\begin{array}{l}6 \\
61 / 8 \\
61 / 4 \\
63 / 8 \\
61 / 2 \\
65 / 8 \\
63 / 4\end{array}$ & $\begin{array}{l}3 / 8 \\
\frac{7}{16} \\
1 / 2 \\
\frac{9}{16} \\
5 / 8 \\
116 \\
8 / 4\end{array}$ & $\begin{array}{l}6.00 \\
6.02 \\
6.06 \\
6.10 \\
6.14 \\
6.18 \\
6.22\end{array}$ & $\begin{array}{l}.25 \\
.27 \\
.31 \\
.35 \\
.39 \\
.43 \\
.47\end{array}$ & $\begin{array}{l}.346 \\
.409 \\
.471 \\
.534 \\
.596 \\
.659 \\
.721\end{array}$ & $\begin{array}{l}.404 \\
.466 \\
.529 \\
.591 \\
.654 \\
.716 \\
.779\end{array}$ & $\begin{array}{l}81 / 2 \\
8 \frac{9}{16} \\
8 \frac{1}{16} \\
8 \frac{1}{16} \\
8 \frac{3}{16} \\
9 \frac{1}{16} \\
9 \frac{3}{16}\end{array}$ & $\begin{array}{l}45 / 8 \\
45 / 8 \\
45 / 8 \\
45 / 8 \\
45 / 8 \\
45 / 8 \\
45 / 8\end{array}$ \\
\hline
\end{tabular}


B ETHLEHEM STEEL COMPANY.

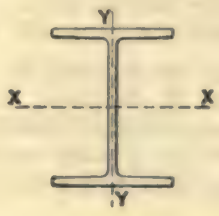

DIMENSIONS AND PROPERTIES OF

BETHLEHEM COLUMNS.

10", 8" AND 6"

- SUPPLEMENTARY SECTIONS.

\begin{tabular}{|c|c|c|c|c|c|c|c|c|c|}
\hline \multirow[b]{2}{*}{$\begin{array}{c}\text { Werght } \\
\text { of } \\
\text { Section, } \\
\text { Lbs. } \\
\text { per } \\
\text { Foot. }\end{array}$} & \multirow[b]{2}{*}{$\begin{array}{l}\text { Area } \\
\text { of } \\
\text { Section } \\
\text { Square } \\
\text { Inches }\end{array}$} & \multicolumn{4}{|c|}{ Axis XX. } & \multicolumn{4}{|c|}{ AXIs YY. } \\
\hline & & $\begin{array}{c}\text { Mo- } \\
\text { ment } \\
\text { of } \\
\text { Inertia. } \\
\text { I } \\
\end{array}$ & $\begin{array}{c}\text { Section } \\
\text { Modu- } \\
\text { lus. } \\
\mathbf{s} \\
\end{array}$ & $\begin{array}{c}\text { Radius } \\
\text { of } \\
\text { Gyra- } \\
\text { tion, } \\
\text { Inches. } \\
\mathbf{r} \\
\end{array}$ & $\begin{array}{c}\begin{array}{c}\text { Bend- } \\
\text { ing } \\
\text { Factor. }\end{array} \\
\mathbf{k} \\
\end{array}$ & $\begin{array}{c}\text { Mo- } \\
\text { ment } \\
\text { of } \\
\text { Inertia. } \\
I^{\prime} \\
\end{array}$ & $\begin{array}{c}\text { Section } \\
\text { Modu- } \\
\text { lus. } \\
\text { s. } \\
\end{array}$ & $\begin{array}{c}\text { Radius } \\
\text { of } \\
\text { Gyra- } \\
\text { tion, } \\
\text { Inches. } \\
\mathbf{r}^{\prime} \\
\end{array}$ & $\begin{array}{c}\text { Bend- } \\
\text { ing } \\
\text { Factor } \\
\mathbf{k}^{\prime} \\
\end{array}$ \\
\hline 33.5 & 9.60 & 166.2 & 34.5 & 4.16 & .278 & 36.6 & 9.14 & 1.95 & 1.050 \\
\hline 38.0 & 10.89 & 192.0 & 39.4 & 4.20 & .276 & 42.4 & 10.56 & 1.97 & 1.031 \\
\hline 42.5 & 12.29 & 219.4 & 44.4 & 4.23 & .277 & 48.5 & 12.02 & 1.99 & 1.022 \\
\hline 47.5 & 13.70 & 247.6 & 49.5 & 4.25 & .277 & 54.8 & 13.52 & 2.00 & 1.013 \\
\hline 23.5 & 6.72 & 74.6 & 19.2 & 3.33 & .349 & 16.8 & 5.17 & 1.58 & 1.298 \\
\hline 27.0 & 7.76 & 88.2 & 22.4 & 3.37 & .347 & 20.0 & 6.11 & 1.60 & 1.270 \\
\hline 30.5 & 8.82 & 102.3 & 25.6 & 3.41 & .345 & 23.2 & 7.07 & 1.62 & 1.248 \\
\hline 34.5 & 9.97 & 117.4 & 28.9 & 3.43 & .345 & 26.6 & 8.07 & 1.63 & 1.236 \\
\hline 20.0 & 5.81 & 38.7 & 12.9 & 2.58 & .451 & 13.0 & 4.34 & 1.50 & 1.340 \\
\hline 23.0 & 6.69 & 45.9 & 15.0 & 2.62 & .446 & 15.4 & 5.12 & 1.52 & 1.305 \\
\hline 26.5 & 7.69 & 53.9 & 17.3 & 2.65 & .445 & 18.1 & 5.96 & 1.53 & 1.290 \\
\hline 30.0 & 8.70 & 62.4 & 19.6 & 2.68 & .445 & 20.8 & 6.82 & 1.55 & 1.277 \\
\hline 33.5 & 9.72 & 71.2 & 21.9 & 2.71 & .444 & 23.6 & 7.69 & 1.56 & 1.264 \\
\hline 37.0 & 10.76 & 80.4 & 24.3 & 2.73 & .443 & 26.6 & 8.59 & 1.57 & 1.251 \\
\hline 40.5 & 11.80 & 90.1 & 26.7 & 2.76 & .442 & 29.6 & 9.52 & 1.58 & 1.240 \\
\hline
\end{tabular}




\section{REINFORCED 14" H COLUMNS \\ AND \\ SPECIAL COMPOUND COLUMNS.}

When columns are required of greater sectional area than the regular sections of $\mathrm{H}$ columns, it is necessary to reinforce the latter to obtain the desired area. This may be the case in the columns for the lower stories of a high building.

Additional area may be secured by riveting plates to the flanges of the regular $\mathrm{H}$ columns. In general this is the simplest and most economical method of reinforcement. Dimensions and properties of such reinforced $14^{\prime \prime} \mathrm{H}$ columns are given on pages 32 and 33 in the table of Reinforced $14^{\prime \prime} \mathrm{H}$ Columns.

If it is desired to avoid drilling the thick metal of the flanges of the heavy $\mathrm{H}$ columns, for attaching the cover plates, the Bethlehem 14" Special H Section, H14 b, shown below, may be used. Dimensions and properties of this special section are given on the opposite page. This special section is produced by the same rolls and has the same inner contour as the regular series of $14^{\prime \prime} \mathrm{H}$ columns on page 18, which can be spliced to this special section in the usual way. This section permits the addition of plates, or other shapes, for increasing the area to the desired extent, in the manner indicated by Figs. 1-3 on the opposite page, avoiding the drilling of thick metal in the flanges. Dimensions and properties of heavy columns, made of this Bethlehem 14" Special H Section with cover plates, are given on pages 34 and 35 in the table of Special Compound Columns.

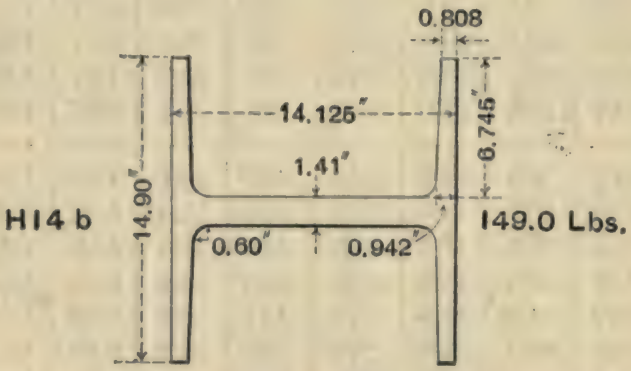


BETHLEHEM STEEL COMPANY.

DIMENSIONS AND PROPERTIES OF

\section{BETHLEHEM 14" SPECIAL H SECTION.}
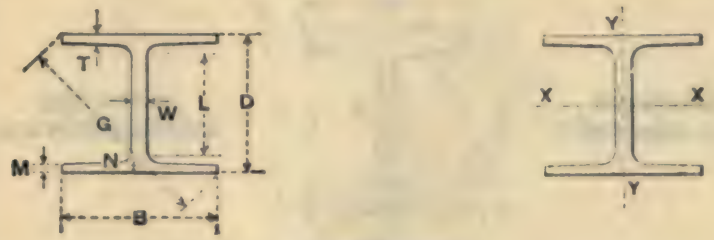

DIMENSIONS.

\begin{tabular}{|c|c|c|c|c|c|c|c|c|c|}
\hline \multirow[b]{2}{*}{$\begin{array}{l}\text { Section } \\
\text { Num- } \\
\text { ber. }\end{array}$} & \multirow{2}{*}{$\begin{array}{l}\text { Weight } \\
\text { of } \\
\text { Section, } \\
\text { Lbs. } \\
\text { per } \\
\text { Foot. }\end{array}$} & \multicolumn{8}{|c|}{ DIMENSIONS, IN INCHES. } \\
\hline & & D & $\begin{array}{l}\text { Nom- } \\
\text { inal. } \\
\qquad \\
\mathbf{T}\end{array}$ & B & W & M & $\mathbf{N}$ & G & L \\
\hline $\mathrm{H14} \mathrm{b}$ & 149.0 & $141 / 8$ & $7 / 8$ & 14.90 & 1.41 & .808 & .942 & $207 / 8$ & $11 \frac{1}{16}$ \\
\hline
\end{tabular}

PROPERTIES.

\begin{tabular}{|c|c|c|c|c|c|c|c|c|}
\hline \multirow[b]{2}{*}{$\begin{array}{l}\text { Section } \\
\text { Number. }\end{array}$} & \multirow[b]{2}{*}{$\begin{array}{l}\text { Weight } \\
\text { of } \\
\text { Section, } \\
\text { Lbs. } \\
\text { per } \\
\text { Foot. }\end{array}$} & \multirow[b]{2}{*}{$\begin{array}{c}\text { Area } \\
\text { of } \\
\text { Section, } \\
\text { Syuare } \\
\text { Inches. }\end{array}$} & \multicolumn{3}{|c|}{ Axis $x x$. } & \multicolumn{3}{|c|}{ AXIS YY. } \\
\hline & & & $\begin{array}{c}\text { Mo- } \\
\text { ment } \\
\text { of } \\
\text { Inertia. } \\
\text { I }\end{array}$ & $\begin{array}{l}\text { Section } \\
\text { Modu- } \\
\text { lus. } \\
\text { S }\end{array}$ & $\begin{array}{c}\text { Radius } \\
\text { of } \\
\text { Gyra- } \\
\text { tion. } \\
\mathbf{r}\end{array}$ & $\begin{array}{l}\text { Mo- } \\
\text { ment } \\
\text { of } \\
\text { Inertia. } \\
\text { I }^{\prime}\end{array}$ & $\begin{array}{l}\text { Section } \\
\text { Modu- } \\
\text { lus. } \\
\mathbf{S}^{\prime}\end{array}$ & $\begin{array}{c}\text { Radius } \\
\text { of } \\
\text { Gyra- } \\
\text { tion. } \\
r^{\prime}\end{array}$ \\
\hline $\mathrm{H} 14 \mathrm{~b}$ & 149.0 & 43.52 & 1368.5 & 193.8 & 5.61 & 468.6 & 629 & 3.28 \\
\hline
\end{tabular}

SUGGESTIONS FOR USING THE BETHLEHEM 14" SPECIAL H SECTION IN BUILDING UP COLUMNS OF LARGE SECTIONAL AREA.

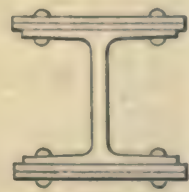

Fig. 1

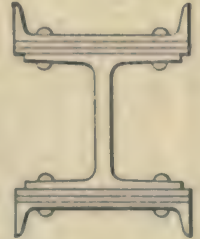

Fig. 2

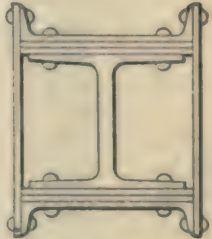

Fig. 3 
BETHLEHEM STEEL COMPANY.

\section{DIMENSIONS AND PROPERTIES OF}

\section{REINFORCED 14" H COLUMNS.}

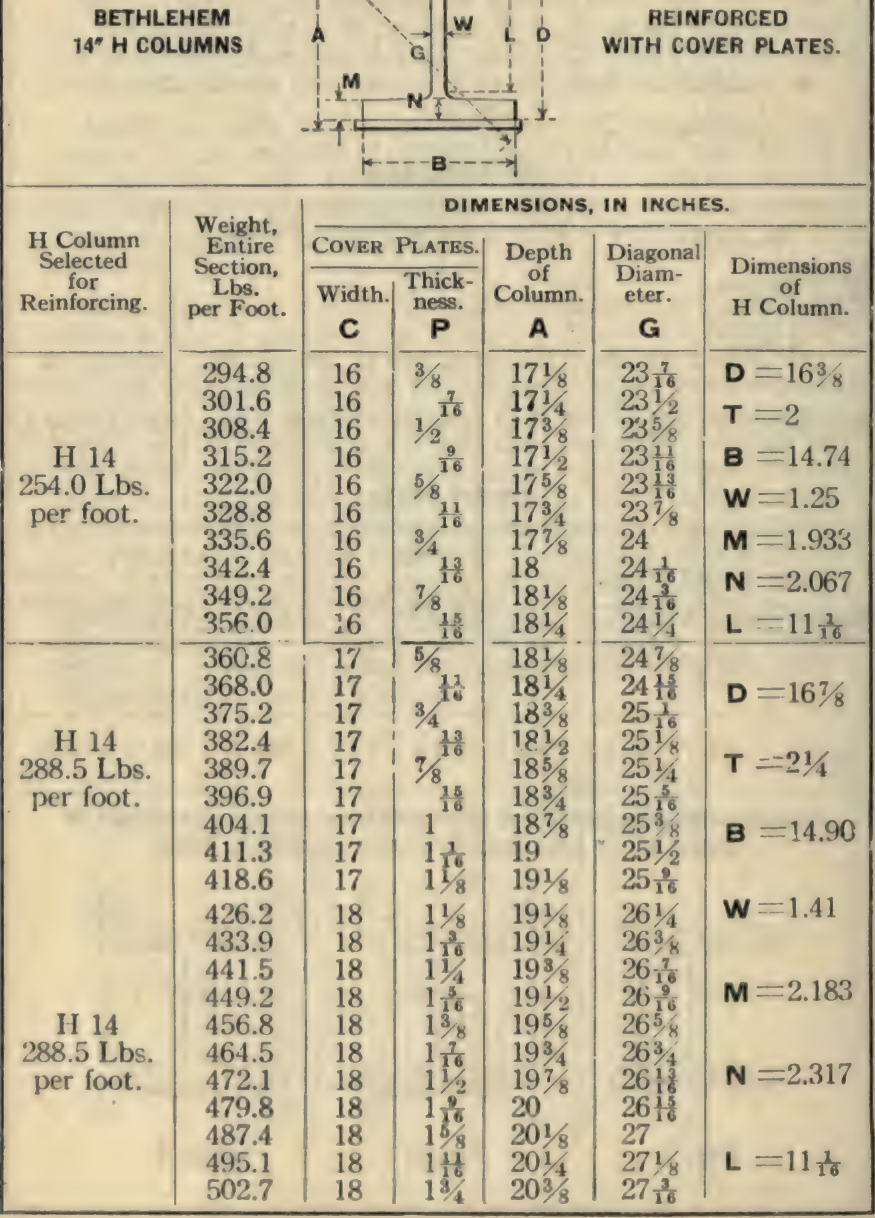


BETHLEHEM STEEL COMPANY.

DIMENSIONS AND PROPERTIES OF

REINFORCED 14" H COLUMNS.

BETHLEHEM
$14^{\prime \prime}$ H COLUMNS

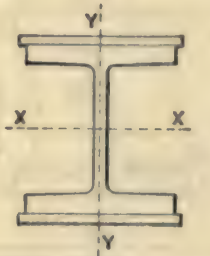

\begin{tabular}{|c|c|c|c|c|c|c|c|c|c|}
\hline \multirow[b]{2}{*}{$\begin{array}{c}\text { Weight, } \\
\text { Encire } \\
\text { Section, } \\
\text { Lbs. } \\
\text { per } \\
\text { Foot. }\end{array}$} & \multirow[b]{2}{*}{$\begin{array}{l}\text { Area, } \\
\text { Entire } \\
\text { Section, } \\
\text { Square } \\
\text { Inches. }\end{array}$} & \multicolumn{4}{|c|}{ AXIS XX. } & \multicolumn{4}{|c|}{ AXIS YY. } \\
\hline & & $\begin{array}{c}\text { Mo- } \\
\text { ment } \\
\text { of } \\
\text { Inertia. } \\
\mathbf{I} \\
\end{array}$ & $\begin{array}{c}\text { Section } \\
\text { Modu- } \\
\text { lus. } \\
\mathbf{s} \\
\end{array}$ & $\begin{array}{c}\text { Radius } \\
\text { of } \\
\text { Gyra- } \\
\text { tion. } \\
\mathbf{r} \\
\end{array}$ & $\begin{array}{c}\begin{array}{c}\text { Bend- } \\
\text { ing } \\
\text { Factor. }\end{array} \\
\mathbf{k} \\
\end{array}$ & $\begin{array}{c}\text { Mo- } \\
\text { ment } \\
\text { of } \\
\text { Inertia. } \\
\mathbf{I}^{\prime} \\
\end{array}$ & $\begin{array}{c}\text { Section } \\
\text { Modu- } \\
\text { lus. } \\
\mathbf{S}^{\prime} \\
\end{array}$ & $\mid \begin{array}{c}\text { Radius } \\
\text { of } \\
\text { Gyra- } \\
\text { tion. } \\
\mathbf{r}^{\prime} \\
\end{array}$ & $\begin{array}{c}\begin{array}{c}\text { Bend- } \\
\text { ing } \\
\text { Factor }\end{array} \\
\mathbf{k}^{\prime}\end{array}$ \\
\hline 24.8 & 86.43 & 410 & 479.4 & 89 & .180 & 1309.2 & 163.6 & 3.89 & .528 \\
\hline & & & & & & & & 3.5 & .523 \\
\hline & 90.43 & & & 6 & 110 & & & 3.93 & .51 \\
\hline & 92.43 & & 520. & 7.0 & .178 & 2 & & 3.94 & .515 \\
\hline 322.0 & 94. & & & 7. & 17 & & & 3 & .510 \\
\hline 28.8 & & & & & .176 & & & & .507 \\
\hline .0 & 98 & 502 & 5 & 1.1 & . & 2 & 1 & 3. & 50.3 \\
\hline 2.4 & 100 & 518 & 576. & 7.1 & .174 & 8 & 20 & 4.00 & .500 \\
\hline & & & & 7. & .17 & & & 4.01 & .496 \\
\hline 6.0 & 10 & & 6 & 7.2 & 1 & 2 & 2 & 4. & . \\
\hline 0.8 & 105 & 54 & 60 & 7 & 175 & $y$ & 20 & 4. & .517 \\
\hline 368.0 & 107 & 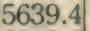 & 6 & 7. & 175 & & & 4.07 & 51 \\
\hline o. & & & & 7. & & & & 9 & \\
\hline & & & & 7. & 1 & & & 1 & \\
\hline 38 & & & & & & & 22 & & 0 \\
\hline 396. & & & 6 & 7 & .1 & 3 & 23 & 4.14 & .496 \\
\hline 404. & 11 & & 6 & 7. & 17 & .5 & 24 & 4. & 492 \\
\hline & & & & & & & & 4 & .489 \\
\hline 418.6 & 122. & 693 & 6 & 7.52 & .169 & & 7 & 4.18 & .486 \\
\hline 6.2 & 12 & & & 7. & 1 & 2320.2 & 25 & 4.31 & 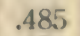 \\
\hline 433. & & & & 7. &. & 2380.9 & 26 & 4.2 & .481 \\
\hline 441 & & & & 7.6 & 166 & & & 4. & .477 \\
\hline & & & & & 16 & & & & .474 \\
\hline & & & & & & & & & .47 \\
\hline & & & & 7 & 1 & & 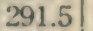 & 4.39 & .46 \\
\hline & & & 845 & 7. & .16 & & 298.3 & 4.40 & .46 \\
\hline 479 & & & & 7.8 & .163 & & & 4.42 & .461 \\
\hline & & & & & & & & & .459 \\
\hline & & & & & & & & & .45 \\
\hline . & & & & 7.95 & & & & 4.46 & .42 \\
\hline
\end{tabular}


BETHLEHEM STEEL COMPANY.

\section{DIMENSIONS AND PROPERTIES OF SPECIAL COMPOUND COLUMNS.}

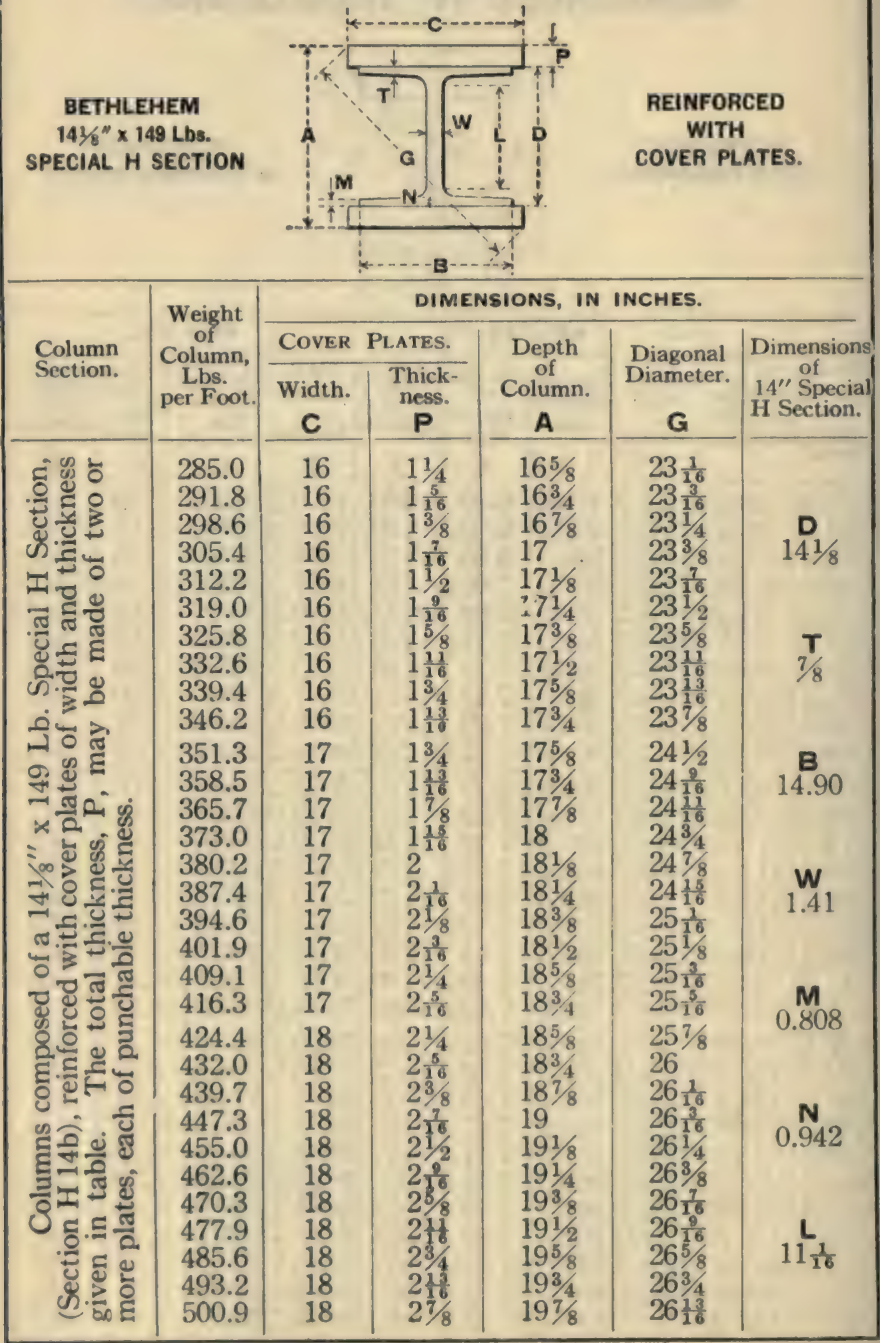




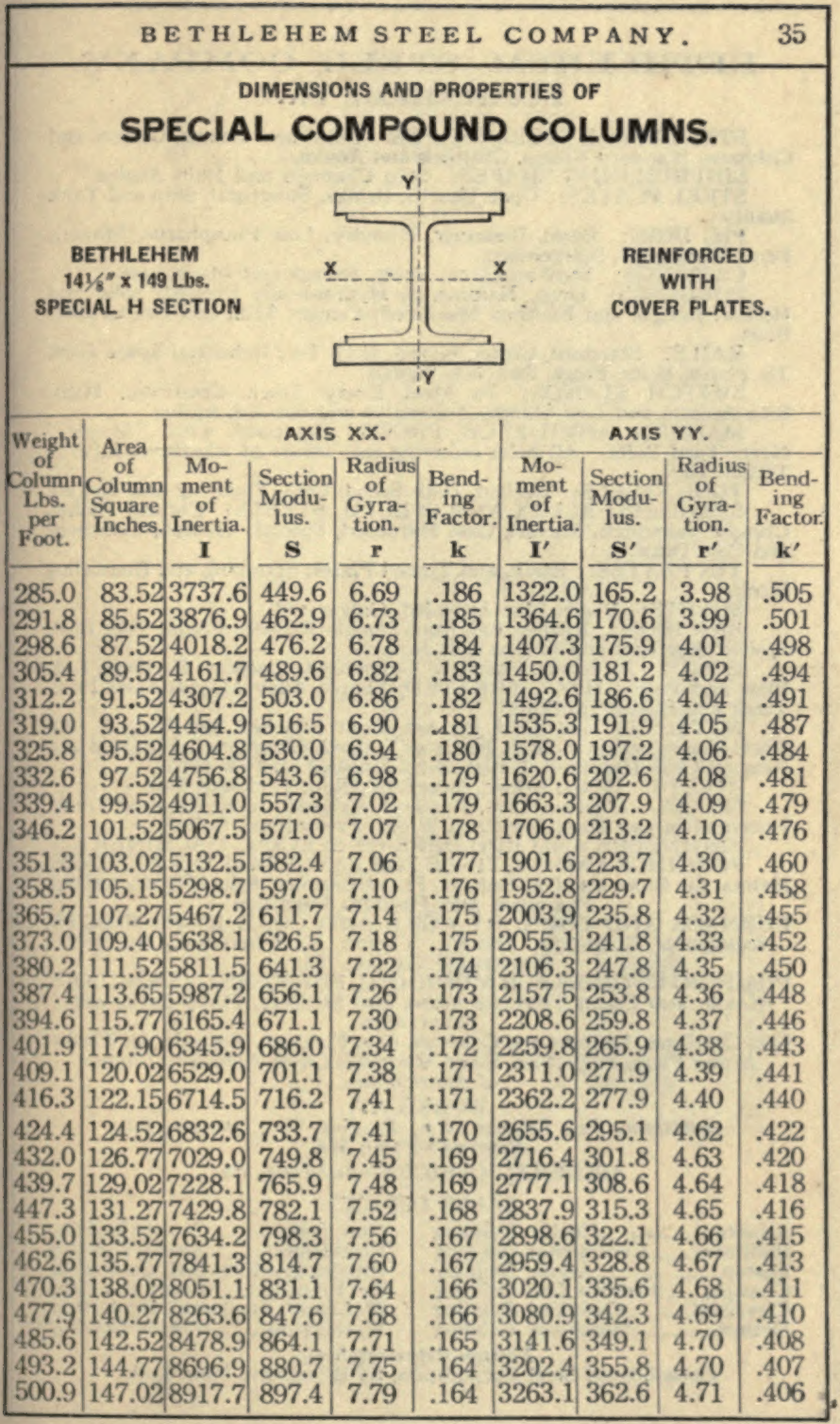




\section{BETHLEHEM STEEL COMPANY BETHIFHEM, PA.}

STRUCTURAL STEEL SHAPES: Bethlehem Beams, Girders and Columns: Standard Beams, Channels and Angles.

SHIPBUILDING SHAPES: Ship Channels and Bulb Angles. quality.

STEEL PLATES: Open Hearth; Bridge, Structural, Ship and Tank

PIG IRON: Basic, Bessemer, Foundry, Low Phosphorus, Mayari, Ferro-Manganese, Spiegeleisen.

CASTINGS: Steel and Iron, Brass, Bronze and Manganese.

FORGINGS: Drop, Hammered, Hydraulically Pressed, Solid and Hollow, Rough, and Finished Machined; Cement Mill, and Ore Crusher Balls.

RAILS: Standard, Girder, Guard, High Tee, Industrial Splice Bars, Tie Plates, Bolts, Frogs, Switches, Signals.

SWITCH STANDS: To Meet Every Track Condition; High, Intermediate and Low Stands, Adjustable and non-Adjustable.

MANARD ANVIL-FACE FROGS: Equipped with "Mayari" Never-Turn Bolts. All bolts receiving side thrust of wheels are HEATTREATED.

TOOL STEELS: Carbon, High Speed, Drill, Finishing and Special. STANDARD ALLOY AND SPECIAL STEELS: Chrome Nickel, Chrome Vanadium, Mayari, Case Hardened, Special; Treated, Tempered and Cold Drawn.

Stock.

TIN PLATES: Black, and Tinned Plates; Stamping and Enameling

SHEETS: Black, Blue-Annealed, Galvanized.

BAR IRON: Refined Bar Iron, Double Refined, Chain, Stay Bolt, Special Stay Bolt and Engine Bolt Iron.

BOLTS: All Kinds, Plain and Galvanized, Machine, Carriage, Lag, Plow and Patch; HEAT TREATED, Simple Alloy Mayari Steel Frog, Track and Fitting-Up Bolts.

NUTS: Hot and Cold Pressed, Cold Punched and Forged. All Sizes, Shapes and Standards, Blank or Tapped, Chamfered, Trimmed and Reamed Case Hardened, Castle, Semi-Finished and Finished.

RIVETS: Boiler, Bridge, Structural, Ship, Tank and Tap.

SPIKES: Steel and Iron, Standard, Railroad, Screw Railroad Track, Universal Screw, Boat, Dock and Wharf, Pressed and Rolled.

ENGINEERS AND BUILDERS OF SPECIAL MACHINERY.

FORGINGS AND CASTINGS FOR LARGE MARINE ENGINES.

Hydraulic Beam and Gap Shears, Plate Bending Rolls, Intensifiers, Flanging and Forging Presses.

FURNACE AND FOUNDRY COKE AND COKE BY-PRODUCTS. AMmonium Sulphats, Benzol, CRude Naphthalene, Solvent NAPHTHAS, TOLUOL, TAR.

PUMPING ENGINES FOR MUNICIPAL PURPOSES,GAS ENGINES. Naval and CoAst Defense GuNs and Mounts, Field GUNS aND HoWITZERS.

Guri Carriages, Limbers and Caissons, Gun Forgings, Torpedo Air Flasks, Armor Plate, Projectiles, Fuses, Cartridge Cases, Complete Rounds of Ammunition.

WORKS AT:

Bethlehem, Pa.; Lebanon, Pa.; Reading, Pa.; Steelton, Pa.; Sparrow's Point, Md.

GENERAL OFFICES-Bethlehem, $P a$.

SALES OFFICES:

Atlanta, .................ealey Building Baltimore,....... Continental Building Boston,................. Oliver Building Chicago,........People's Gas Building Cleveland,........... Guardian Building Detroit,.............Penobscot Building

New York. Philadelphia,.......... Morris Building Pittsburgh,... First Nat. Bank Bldg. San Francisco,..... Monadnock Bldg. St. Louis,....Boatmen's Bank Bldg. Washington,........ Wilkins Building Foreign Representatives:

Consolidated Steel Corporation, 25 Broadway, New York. 


\section{PLEASE DO NOT REMOVE \\ CARDS OR SLIPS FROM THIS POCKET}

\section{UNIVERSITY OF TORONTO LIBRARY}

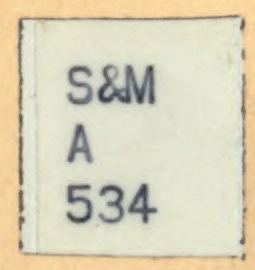


